\title{
EXPLORING THE EFFECTS OF STELLAR ROTATION AND WIND CLEARING: DEBRIS DISKS AROUND F STARS
}

\author{
Trisha F. Mizusawa ${ }^{1,6}$, Luisa M. Rebull ${ }^{2}$, John R. Stauffer $^{2}$, Geoffrey Bryden $^{3,6}$, Michael Meyer $^{4}$, And Inseok Song ${ }^{5}$ \\ ${ }^{1}$ Florida Institute of Technology, 150 W. University Boulevard, Melbourne, FL 32901, USA; trisha.mizusawa@ gmail.com \\ ${ }^{2}$ Spitzer Science Center (SSC), 1200 E. California Blvd., California Institute of Technology, Pasadena, CA 91125, USA \\ ${ }^{3}$ Jet Propulsion Laboratory, 4800 Oak Grove Blvd, Pasadena, CA 91109, USA \\ ${ }^{4}$ ETH, Zurich, Switzerland \\ ${ }^{5}$ Department of Physics and Astronomy, University of Georgia, Athens, GA 30602, USA \\ Received 2012 February 15; accepted 2012 August 29; published 2012 October 2
}

\begin{abstract}
We have conducted a study of debris disks around F stars in order to explore correlations between rotation, stellar winds, and circumstellar disks. We obtained new $24 \mu \mathrm{m}$ photometry from the Multiband Imaging Photometer for Spitzer (MIPS) camera for a sample of 188 relatively nearby F dwarfs with various rotation rates and optical colors, and combined it with archival MIPS data for 66 more F stars, as well as Wide-field Infrared Survey Explorer data for the entire sample, plus 9 more F stars. Based on the objects' $K_{s}-$ [24] and [3.4] - [22] colors, we identify 22 stars in our sample as having 22 and/or $24 \mu \mathrm{m}$ excesses above our detection limit, 13 of which are new discoveries. Our overall disk detection rate is $22 / 263$, or $8 \%$, consistent with previous determinations of disk fractions in the solar neighborhood. While fast-rotating stars are expected to have strong winds capable of efficiently removing dust, we find no correlation between rotational velocity and infrared excess. Similarly, we find no significant difference in excess detection rate between late-type F stars, which have convective surfaces, and early-type F stars, which have fully radiative envelopes. However, the essentially unknown range of ages in this sample may be washing out any effects relating rotation, winds, and disks.
\end{abstract}

Key words: circumstellar matter - infrared: stars

Online-only material: color figures, machine-readable and VO tables

\section{INTRODUCTION}

Infrared Astronomy Satellite and Spitzer Space Telescope (Werner et al. 2004) observations of field A stars and of open clusters have shown that debris disks are common at early ages and decrease in both frequency and luminosity for older stars. Based on images from the Multiband Imaging Photometer for Spitzer (MIPS; Rieke et al. 2004), Su et al. (2006) found about a $30 \%$ frequency of such disks at $24 \mu \mathrm{m}$ for a sample of $<1$ Gyr old A stars, with the upper envelope to the $24 \mu \mathrm{m}$ excess flux decaying roughly as $t_{0} / t$, with $t_{0} \sim 150 \mathrm{Myr}$. Studies of a number of open clusters (e.g., Currie et al. 2009 and references therein) agree with the general trends found for the field A dwarfs.

The debris disk frequency for low-mass stars is less well determined, but again observations of field stars (e.g., Carpenter et al. 2009) and of open clusters (e.g., Rebull et al. 2008 and references therein) indicate that the debris disk frequency and luminosity peaks at young ages and declines after that, with few or no debris disks detected at $24 \mu \mathrm{m}$ by Hyades or Praesepe age ( 650 Myr; Cieza et al. 2008; Urban et al. 2012).

For the relatively luminous A stars, radiation pressure removes dust particles from the disk on timescales $\ll 1 \mathrm{Myr}$; the dust in these debris disks must be constantly resupplied from collisional events among planetesimals. The lower luminosities of late-type (GKM) dwarfs are less effective in removing the dust via blowout or traditional (radiative) Poynting-Robertson drag. However, as described by Chen et al. (2005) and Plavchan et al. $(2005,2009)$, winds from rapidly rotating, low-mass stars should engender a particulate Poynting-Robertson effect that is more efficient at removing orbiting dust.

\footnotetext{
6 Work conducted while resident at NASA Star and Exoplanet Database (NStED), 1200 E. California Blvd., California Institute of Technology, Pasadena, CA 91125, USA.
}

F stars inhabit a sweet spot between the realms of more massive and less massive stars. It is within the F spectral type where outer convective envelopes begin to appear, at spectral type F5. Higher mass stars have convective cores and radiative envelopes, and winds from the photonic Poynting-Robertson effect scour dust from circumstellar disks. Lower mass stars have strong dynamo activity caused by convective motions and magnetic fields in their outer convective envelopes; strong winds should scour dust from circumstellar disks. As shown by Simon \& Landsman (1991), Wolff \& Simon (1997), and others, there is good evidence that the transition to strong, dynamo-driven winds occurs for stars later than F5-with activity indicators such as $\mathrm{Ca}$ II $\mathrm{HK}$ emission correlated with rotation for later-type stars, and not correlated for earlier-type stars.

It has long been known that the $v \sin i$ distribution of mainsequence (MS) stars shows a transition at spectral-type F. Earlytype $(\mathrm{O}, \mathrm{B}$, and $\mathrm{A})$ stars are generally rapid rotators, with average rotational velocities of $200-300 \mathrm{~km} \mathrm{~s}^{-1}$. MS G, K, and $\mathrm{M}$ dwarfs (older than a few hundred Myr) are generally very slow rotators, with mean rotational velocities of only a few $\mathrm{km} \mathrm{s}^{-1}$. There is thus a sharp drop in the mean rotation rate of MS stars as one goes from F0 to F9. Interestingly, when this facet of rotation on the MS was first recognized, two possible explanations were advanced-angular momentum loss via winds (Mestel \& Roxburgh 1962) and angular momentum redistribution into planetary systems (Huang 1965). The former explanation was eventually recognized as the primary physical mechanism responsible for the drop in rotational velocities.

However, the strength of those winds-or at least the amount of angular momentum they carry away-is a subject of controversy. Some models (e.g., Denissenkov et al. 2010; Sills et al. 2000) predict that only the outer convective envelope is spun down (initially) by the wind; because the amount of mass (and the moment of inertia) present in the outer convective 
envelope of late F stars is very small, in this event the inferred mass loss rate could be small even for rapidly rotating $\mathrm{F}$ stars. Other models, however, predict strong coupling between the convective envelope and the radiative interior, and postulate that the entire star is spun down on short timescales even for late F stars (Bouvier et al. 1997). The inferred mass-loss rate must be much higher in this event.

Because of the rapid change of wind properties through the F star regime, stars of these masses provide unique laboratories for studies of wind-related effects. Debris disks could be more common around F stars near the transition between early and late F stars, and could persist for longer periods than for either higher or lower mass stars (and may result in planets with high obliquities; see Winn et al. 2010). However, for stars with massive disks, the effect of PR drag may be small compared to mutual collisions of dust within a disk such that the grains are ground down into fine enough particles that radiation pressure can remove the dust (e.g., Wyatt 2005).

We have conducted a MIPS $24 \mu \mathrm{m}$ survey of 254 field $\mathrm{F}$ dwarfs, half of which rotate quickly $\left(>15 \mathrm{~km} \mathrm{~s}^{-1}\right)$, the other half of which rotate slowly $\left(\leqslant 15 \mathrm{~km} \mathrm{~s}^{-1}\right)$. We added to the sample nine objects detected in Wide-field Infrared Survey Explorer (WISE) but missing from the MIPS archive. Our goal was to determine if (1) $\mathrm{F}$ dwarfs have a comparatively high debris disk frequency and (2) we see evidence (via debris disk frequency) for development of winds at the F5 boundary where solar-type winds are expected to develop. Section 2 presents the data analysis and reduction, Section 3 identifies infrared excesses, and Section 4 discusses the results from this paper.

\section{MIPS $24 \mu \mathrm{m}$ PHOTOMETRY AND DATA ANALYSIS}

\subsection{Target Selection}

We merged the Hipparcos catalog with other catalogs containing spectroscopic rotational velocities (see below). We selected all stars with spectral types F0 to F9 and distances less than $100 \mathrm{pc}$, and required that the targets were above (or below) a galactic latitude of $9^{\circ}\left(|b|>9^{\circ}\right)$ and had an ecliptic latitude above $19^{\circ}\left(|\beta|>19^{\circ}\right)$. This resulted in $\sim 7000$ stars. In order to reinforce the spectral type restriction, we retained stars with $0.30>B-V>0.52$. To retain only MS stars and mitigate somewhat against binaries, we eliminated stars more than 0.1 mag fainter than the single-star ZAMS (zero-age main sequence) or brighter than 0.6 mag above the ZAMS. Because we were interested in correlations with rotation, we only retained objects for which there were measurements of $v \sin i$. We split the sample into a blue set $(0.30 \leqslant B-V \leqslant 0.41)$ and a red set $(0.43 \leqslant B-V \leqslant 0.52)$. Note that there is a clear and artificially imposed distinction in color for these two samples; we dropped stars with $B-V$ colors between 0.41 and 0.43 (e.g., colors centered on F5) to make the samples more cleanly and explicitly divided into early and late $\mathrm{F}$ (radiative and convective envelopes, respectively). Each of these sets was again bifurcated into a slowly rotating set and a rapidly rotating set $(v \sin i \leqslant$ or $>15 \mathrm{~km} \mathrm{~s}^{-1}$ ). This division between fast and slow rotation was selected because the X-ray flux saturates for G stars at about $15 \mathrm{~km} \mathrm{~s}^{-1}$; we assume that wind strength and X-ray flux are roughly correlated. Lacking other guidance, we extrapolated that limit to the F star regime. Each group of remaining F stars was then sorted by distance, which we then compared with the objects already observed by Spitzer. We requested new Spitzer observations of the $\sim 50$ closest objects not yet observed in each group.
Our target selection for new observations by definition deliberately sought objects that were not already in the Spitzer archive. There is some reason to believe that observations already obtained by others in the Spitzer archive may be biased toward objects with disks-for example, Moór et al. (2011) who deliberately observed F stars thought to have disks, or Rebull et al. (2008) who deliberately observed stars thought to be members of the Beta Pic Moving Group (which includes an $\mathrm{F}$ star, and stars belonging to this moving group are young enough that they are still likely to have substantial disks). We must include in our analysis objects whose observations were already in the Spitzer archive, but we do so knowing that those archive observations may not have been taken with the same sensitivity as our new observations. All of our targets are bright (easily detected), so this is not likely to introduce any biases, per se. However, there were considerably more red (late) F stars already in the Spitzer archive than blue (early) F stars (49/156 versus $18 / 109)$, so when the entire sample is considered, the net number of late F stars in the Spitzer archive (our new $\sim 100$ observations of red $\mathrm{F}$ stars plus that of others, a total of 156 objects) is greater than the number of early F stars (a total of 109 objects). It is also true that the late F stars are on average closer to us than the early F stars. As a result, our blue sample reaches out to greater distances $(96 \mathrm{pc})$ than the red sample (67 pc).

Subsequent to our original proposal, the all-sky catalog from WISE (Wright et al. 2010) has been released. WISE surveyed the whole sky in four bands, 3.4, 4.6, 12, and $22 \mu \mathrm{m}$. WISE is very different from MIPS - the WISE $22 \mu \mathrm{m}$ bandpass is distinctly different from the MIPS $24 \mu \mathrm{m}$ bandpass, the spatial resolution is twice as good with MIPS $\left(\sim 6^{\prime \prime}\right)$ as with WISE $\left(\sim 12^{\prime \prime}\right)$, and the sensitivity is in general better with MIPS than with WISE (where the sensitivity varies with ecliptic latitude). However, since WISE observed the whole sky, and since our targets are generally bright, we can use the WISE data to complement our sample. For example, we can use the [3.4]-[22] (W1-W4) color as an independent check on the IR excess we determine using $K_{s}-[24]$. In some cases, we can include objects that were dropped from our Spitzer target selection because we thought they were in the Spitzer archive, but the objects were just off the edge of the observations.

We selected targets for our sample (for our new observations, or from the Spitzer archive, or from WISE) without particular bias toward the presence or absence of disks. Because we started from many different catalogs aimed at studies of nearby stars, there should be no particular biases imposed by, e.g., requiring $v \sin i$ any more than the requirement that there be a good $B$ and $V$ measurement. However, as will be seen below, we probably have an implicit bias in that the rapid rotators are more likely to be young.

The final set of stars is shown in an $M_{v}$ versus $B-V$ color-magnitude diagram (CMD) in Figure 1 (and listed in Table 1; also see Table 3 for relevant notes about certain stars). The visible gap between the blue and red samples in Figure 1 is an explicit result of the selection process above; this gap corresponds to the F5 division between stars with radiative and convective envelopes. We could not identify any statistically significant difference between Figure 1 distribution of the bluerapid and blue-slow stars, or in the distribution of red-rapid and red-slow stars, which would imply similar age distributions. However, there is an expectation that the rapid rotators should be younger than the slow rotators, on average, and this effect should be more pronounced for the red sample; we will discuss 


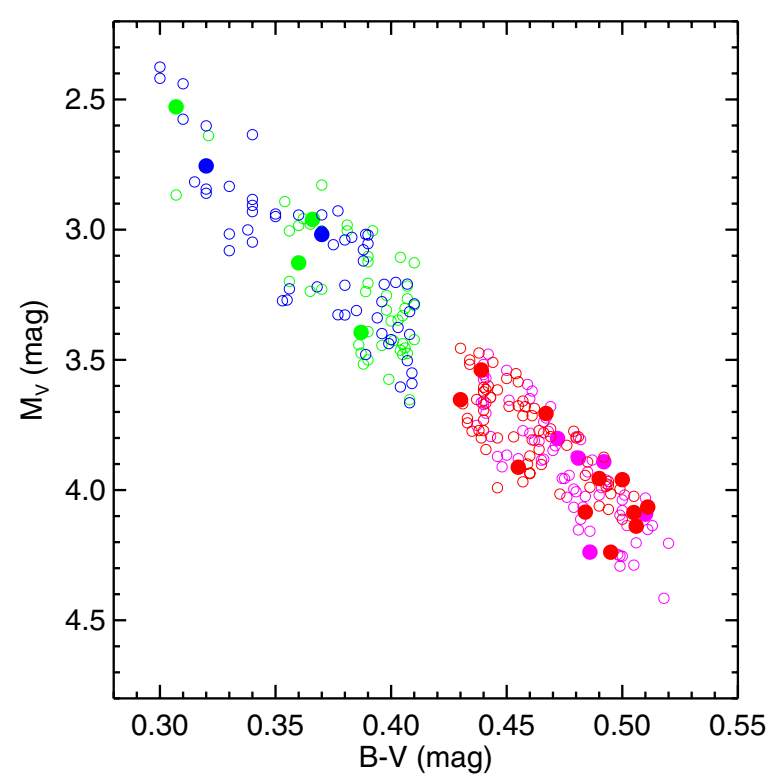

Figure 1. $M_{v}$ vs. $B-V$ for the full sample of stars, which shows the as-designed gap in colors near $B-V \sim 0.42$, or type F5. Green circles represent the blue-slow group, blue circles represent the blue-rapid groups, magenta circles represent the red-slow group, and red circles represent the red-rapid group. Filled symbols indicate infrared excess stars (see Section 3). In this figure, there is no statistical difference between the distributions of either of the red groups or either of the blue groups, suggesting that the stars span the same range of ages. However, the age range may be significant; see the discussion in Section 4.

this in more detail below. Any ages assumed from the $M_{v} / B-V$ CMD are unlikely to be very accurate.

\subsection{Observations}

Each of the 188 targets observed as part of our original Cycle-5 program 50449 (PI: L. Rebull) was observed using a MIPS photometry AOR (Astronomical Observation Request) at $24 \mu \mathrm{m}$. Our AORs are the same for all of our targets, using one cycle of $3 \mathrm{~s}$ integration time at $24 \mu \mathrm{m}$ for a total integration time of $42 \mathrm{~s} \mathrm{pixel}^{-1}$ on-target. There are 69 more objects for which MIPS-24 observations were obtained external to program 50449. While most of these were MIPS photometry AORs targeting our object of interest, many were not, and our object of interest was caught serendipitously in a MIPS scan observation, or a MIPS photometry observation of another target. Some were observed in more than one AOR.

For each observation, we extracted the individual basic calibrated data (BCD) files (individual flux density calibrated array images), or enhanced BCD files, as appropriate, and pipelinecreated ("post-BCD") mosaics from the Spitzer Science Center (SSC) Spitzer Heritage Archive (SHA) which were created using pipeline version S18.13. We investigated each mosaic for instrumental artifacts such as jailbars for bright sources or dark latents from bright objects observed prior to our observations. All of the mosaics were sufficiently clear of artifacts at the position of our source of interest, so we used the pipeline-produced mosaics for our targets. Our targets are bright, but none were saturated.

\subsection{Photometry}

For each of the final $\sim 260$ MIPS-24 mosaics, we used the APEX-1Frame package from MOPEX (Makovoz \& Marleau 2005) to perform point-response function (PRF) fitting to obtain photometry for each object using the PRFs provided on the
SSC Web site, again using the standard SSC-recommended set of parameters. At the same time, we also used APEX to perform aperture photometry on the targets, as a check on the flux densities we obtained from PRF fitting, to make sure that the results were consistent. For the aperture photometry, we used an aperture radius of $13^{\prime \prime}$ and an annulus of $20^{\prime \prime}-32^{\prime \prime}$, and the corresponding aperture correction of 1.17 from the MIPS Instrument Handbook on the SSC Web site. With these choices, we find that the PRF and aperture photometry yield consistent results, with the mean difference being $\langle\mathrm{PRF}-$ aperture $\rangle=$ $0.030 \pm 0.007 \mathrm{mag}$.

In four cases, nearby objects are at risk of compromising the MIPS-24 photometry, which we now discuss. HIP 9727 is a visual binary, but it is also very bright. APEX erroneously identified two sources at the location of the target (which is known to happen for very bright objects such as this target); we confirmed using point-spread-function (PSF) subtraction (module apex_qa) that there are unlikely to be two sources cleanly detected by APEX. In that case, the aperture photometry was used instead of the PRF-fitting photometry. (We do not identify this object as having an IR excess $-K_{s}-[24]=0.04$, $[3.4]-[22]=0.01$.)

For two objects, HIP 105547 and HIP 99680, APEX identified more than one source within $\sim 5^{\prime \prime} .5$ of the desired target. Using PSF subtraction (module apex_qa), it is clear that both sources are legitimately detected in both mosaics. The PRF-fitted flux density at $24 \mu \mathrm{m}$ is used for these two sources, and the WISE measurements are tagged as unreliable, as WISE is unlikely to be unable to resolve each of these pairs of objects (e.g., $\chi_{\text {best }}=\chi_{24}$; see below). We note that HIP 105547 is reported in the literature as a binary, but HIP 99680 is not.

In the last case, HIP 32435, there are MIPS data in the SHA, but by looking at the image, we determined that this object seemed to be confused with a background galaxy. We dropped this object from our sample entirely, as determining a flux density from the star alone would be difficult (using MIPS or WISE).

In a few observations of objects pulled out of the archive, where our object of interest was caught serendipitously, sometimes the object fell in a region with less-than-full coverage (HIP 19480, HIP 67103, HIP 72603, HIP 78547). Since our objects are bright, even if there were fewer MIPS frames than "normal" for most of the observation, often adequate photometry could be obtained. We do not identify any of these objects as having an IR excess.

In some of the observations pulled out of the archive, more than one observation was obtained of our target (HIP 1427, HIP 3961, HIP 20491, HIP 27633, HIP 66121). In these cases, we measured photometry independently on the two (or three) pipeline mosaics (one is created per AOR in the pipeline) and took a weighted average as the final best value for the $24 \mu \mathrm{m}$ flux density. Just one of these objects is included in the objects we identify as having an IR excess: HIP 1427.

APEX provides the internal statistical uncertainties on the $24 \mu \mathrm{m}$ flux densities, and are $\sim 1 \%$ or less. We have added a $2 \%$ systematic calibration uncertainty (Engelbracht et al. 2007; J. E. Gizis 2009, private communication) on top of this; this is added in quadrature to the errors returned by APEX to obtain the errors we used. We converted the measured $24 \mu \mathrm{m}$ flux densities and uncertainties using the zero-point magnitude of $7.17 \mathrm{Jy}$, from the SSC Web site (derived by Rieke et al. 2008). 


\begin{tabular}{|c|c|c|c|c|c|c|c|c|c|c|c|c|c|c|c|}
\hline Name & $\begin{array}{l}\text { Distance } \\
\text { (pc) }\end{array}$ & $\begin{array}{c}V \\
(\mathrm{mag})\end{array}$ & $\begin{array}{c}B-V \\
(\mathrm{mag})\end{array}$ & $\begin{array}{c}v \sin i \\
\left(\mathrm{~km} \mathrm{~s}^{-1}\right)\end{array}$ & Sample & $\begin{array}{c}K_{s} \\
(\mathrm{mag})\end{array}$ & AORKEY(s) & $\begin{array}{c}{[24]} \\
(\mathrm{mag})\end{array}$ & $\begin{array}{c}K_{s}-[24] \\
(\mathrm{mag})\end{array}$ & $\chi_{24}$ & $\begin{array}{c}{[3.4](\mathrm{W} 1)} \\
(\mathrm{mag})\end{array}$ & $\begin{array}{c}{[22](\mathrm{W} 4)} \\
(\mathrm{mag})\end{array}$ & $\begin{array}{c}{[3.4]-[22]} \\
\quad(\mathrm{mag})\end{array}$ & $\chi_{22}$ & $\chi_{\text {best }}$ \\
\hline HIP 462 & 50 & 7.13 & 0.45 & 24 & Red-rapid & $6.01 \pm 0.02$ & 17346304 & $6.017 \pm 0.022$ & $-0.010 \pm 0.030$ & 0.65 & $5.95 \pm 0.05$ & $5.86 \pm 0.04$ & $0.087 \pm 0.066$ & 1.32 & 1.14 \\
\hline HIP 606 & 48 & 7.05 & 0.46 & 9 & Red-slow & $5.91 \pm 0.02$ & 26198784 & $5.930 \pm 0.022$ & $-0.015 \pm 0.029$ & 0.52 & $5.86 \pm 0.05$ & $5.88 \pm 0.04$ & $-0.021 \pm 0.066$ & -0.32 & 0.35 \\
\hline HIP 950 & 21 & 5.25 & 0.44 & 5 & Red-slow & $4.13 \pm 0.31$ & 10234112 & $4.178 \pm 0.022$ & $-0.044 \pm 0.307$ & -0.05 & $4.12 \pm 0.09$ & $4.09 \pm 0.02$ & $0.029 \pm 0.095$ & 0.31 & 0.28 \\
\hline HIP 1134 & 46 & 7.32 & 0.50 & 37 & Red-rapid & $6.07 \pm 0.02$ & 5272064 & $6.102 \pm 0.022$ & $-0.029 \pm 0.030$ & 0.04 & $6.05 \pm 0.05$ & $6.02 \pm 0.05$ & $0.026 \pm 0.066$ & 0.40 & 0.20 \\
\hline HIP 1402 & 48 & 6.45 & 0.37 & 8 & Blue-slow & $5.43 \pm 0.02$ & 26213888 & $5.464 \pm 0.022$ & $-0.029 \pm 0.028$ & 0.04 & $5.40 \pm 0.06$ & $5.37 \pm 0.03$ & $0.035 \pm 0.072$ & 0.48 & 0.21 \\
\hline HIP 1427 & 46 & 7.20 & 0.48 & 13 & Red-slow & $6.05 \pm 0.02$ & $17951744,17951232,17951488$ & $5.931 \pm 0.022$ & $0.121 \pm 0.028$ & 5.49 & $6.04 \pm 0.05$ & $5.90 \pm 0.05$ & $0.140 \pm 0.067$ & 2.08 & 5.87 \\
\hline HIP 2337 & 42 & 6.14 & 0.37 & 40 & Blue-rapid & $5.20 \pm 0.02$ & 26205184 & $5.218 \pm 0.022$ & $-0.022 \pm 0.030$ & 0.26 & $5.22 \pm 0.07$ & $5.14 \pm 0.03$ & $0.074 \pm 0.079$ & 0.94 & 0.58 \\
\hline HIP 3214 & 57 & 7.59 & 0.44 & 30 & Red-rapid & $6.51 \pm 0.02$ & 26180608 & $6.499 \pm 0.023$ & $0.007 \pm 0.029$ & 1.26 & $6.47 \pm 0.04$ & $6.46 \pm 0.05$ & $0.010 \pm 0.064$ & 0.16 & 1.22 \\
\hline HIP 3505 & 27 & 5.24 & 0.33 & 42 & Blue-rapid & $4.36 \pm 0.02$ & 26202368 & $4.355 \pm 0.022$ & $0.004 \pm 0.027$ & 1.27 & $5.12 \pm 0.02$ & $4.22 \pm 0.02$ & $0.899 \pm 0.033$ & 27.64 & 1.27 \\
\hline HIP 3641 & 40 & 6.39 & 0.40 & 15 & Blue-slow & $5.35 \pm 0.02$ & 26221824 & $5.361 \pm 0.022$ & $-0.008 \pm 0.030$ & 0.71 & $5.31 \pm 0.06$ & $5.29 \pm 0.03$ & $0.014 \pm 0.072$ & 0.20 & 0.73 \\
\hline
\end{tabular}

(This table is available in its entirety in machine-readable and Virtual Observatory (VO) forms in the online journal. A portion is shown here for guidance regarding its form and content.) 
Table 2

Bright Sources

\begin{tabular}{lcccr}
\hline \hline Name & $K_{s}$ from 2MASS & $K_{s}$ Used Here & $K_{s}-[24]$ & {$[3.4]-[22]$} \\
\hline HIP 16245 & 3.936 & 3.79 & 0.02 & 0.00 \\
HIP 21770 & 3.724 & 3.64 & 0.06 & 0.06 \\
HIP 22449 & 1.600 & 2.11 & -0.10 & 0.22 \\
HIP 28103 & 2.993 & 2.90 & 0.04 & 0.13 \\
HIP 33202 & 3.888 & 3.91 & 0.01 & -0.07 \\
HIP 34834 & 3.320 & 3.67 & 0.03 & 0.07 \\
HIP 36366 & 2.978 & 3.39 & 0.13 & -0.01 \\
HIP 38423 & 3.841 & 3.96 & 0.11 & -0.02 \\
HIP 40843 & 3.868 & 4.02 & 0.08 & 0.04 \\
HIP 50954 & 3.252 & 3.14 & 0.02 & 0.02 \\
HIP 61174 & 3.372 & 3.34 & 0.54 & 0.67 \\
HIP 63503 & 3.953 & 4.06 & 0.09 & 0.08 \\
HIP 64241 & 3.108 & 3.29 & 0.04 & 0.03 \\
HIP 73996 & 3.863 & 3.87 & -0.02 & 0.07 \\
HIP 76829 & 3.802 & 3.71 & 0.12 & 0.07 \\
HIP 80179 & 4.094 & 4.01 & 0.00 & 0.01 \\
HIP 82860 & 3.736 & 3.75 & 0.13 & 0.03 \\
HIP 96441 & 3.537 & 3.57 & 0.04 & 0.09 \\
\hline
\end{tabular}

The stars and their AORKEYs are displayed in Table 1, along with the $24 \mu \mathrm{m}$ and the supporting photometry obtained from the literature (see the next section).

\subsection{Supporting Data}

\subsubsection{Optical and NIR Data}

Ancillary optical data were primarily obtained from the merging of the Hipparcos catalog with the literature. The $B$ and $V$ photometry comes from Tycho-2, where the Bessell (2000) formula was used to convert $B_{T}-V_{T}$ to $(B-V)$ on the Johnson system. The $J H K_{s}$ photometry is from the Two Micron All Sky Survey (2MASS; Skrutskie et al. 2006). Below, we will be using $K_{s}-[24]$ as one indicator of excess; we chose to use this rather than $V-[24]$ because of the uniformity and consistency of values available via 2MASS for $K_{s}$. Rotational velocity measurements mostly come from Nordstrom et al. (2004), though some come from Holmberg et al. (2007) or Schröder et al. (2009). In our catalog, $v \sin i \leqslant 3 \mathrm{~km} \mathrm{~s}^{-1}$ should be interpreted as an upper limit. Operationally, we treat the upper limits as detections because whether the rotational velocity is $3 \mathrm{~km} \mathrm{~s}^{-1}$ or $<3 \mathrm{~km} \mathrm{~s}^{-1}$ does not matter since they are all slow rotators.

\subsubsection{Objects with Very Bright or Incorrect $K_{s}$}

2MASS, as reported in the documentation (Cutri et al. 2003; Huchra et al. 1994), does not acquire particularly reliable photometry due to saturation for stars with $K_{s}<3.5$, and stars with $K_{s}<4.0$ often have large errors. Quite a few stars in our sample (18) are in this regime, and we now describe how we obtained more accurate $K_{s}$ estimates for these stars. The resulting $K_{s}$ magnitudes for these stars, with their respective $K_{s}-$ [24] and [3.4]-[22] colors, can be found in Table 2. (Table 1 also contains the $K_{s}$ values we used.)

The brightest object in our sample is HIP 22449, which has a $K_{s}$ in 2MASS reported as 1.6. Additional literature estimates could bring it perhaps to $\sim 2$ mag. Its brightness as measured in [24], [3.4], and [22] are all $\sim 2$ mag. Using the best possible $K_{s}$ we have, $K_{s}-[24]=-0.14$ and [3.4] $-[22]=0.22$. This object could have an IR excess based on the WISE color, though it is so bright that it is often used as a standard in the literature, and no one to date has reported indications of a disk around it. We drop this object from consideration in our sample because it is so bright that our ability to obtain high-confidence estimates of its brightness is limited.

For the remaining bright stars, we estimate $K_{s}$ from the published $V$ and $B-V$ photometry and a relation between $B-V$ and $V-K_{s}$ derived from high probability members of the Praesepe open cluster. Praesepe is near enough that its F stars have high-quality optical photometry, but far enough away that those same stars have good 2MASS $K_{s}$ magnitudes. The relation derived from the Praesepe stars is $\left(V-K_{S}\right)=$ $2.219(B-V)+0.07$. The rms of the Praesepe stars about that relation is 0.059 , which we take to be the uncertainty in the derived $K_{s}$ (compared to stars with directly measured $K_{s}$ ).

The known excess star HIP 61174 is the only star in this group with a 22 or $24 \mu \mathrm{m}$ excess; it has $K_{s}-[24]=0.54$ and $[3.4]-[22]=0.67$.

HIP 8805 is not particularly bright, but is a visual binary in the blue, slowly rotating group, with a separation of about 3 ". 8 . The Hipparcos and Tycho-2 data suggest $V \sim 7.51$ and $8.14 \mathrm{mag}$, and $B-V \sim 0.38$ and $0.52 \mathrm{mag}$, for components $\mathrm{A}$ and $\mathrm{B}$, respectively. The $2 \mathrm{MASS}$ point-source catalog (PSC) claims to provide photometry for both components, but the listed values seem too bright and too similar $\left(K_{s}=6.227\right.$ and $6.280 \mathrm{mag}$, respectively). We removed this star from our analysis because of the unreliable near-IR photometry, but still provide the data in Table 1 for reference. Using the data as provided in Table 1, this star has one of the bluest $K_{s}-$ [24] values of $-0.40 \mathrm{mag}$, lending credence to our suspicion that something is wrong with the $K_{s}$ photometry. It has a very small WISE color, [3.4]-[22] = $0.05 \mathrm{mag}$, so we suspect that this object does not actually have an IR excess or a disk. We have left it in the sample as a WISE-only source (e.g., $\chi_{\text {best }}=\chi_{22}$; see below), and have not identified it as having a disk.

\subsubsection{Objects with Large $K_{s}$ Errors}

Four objects (HIP 9782, HIP 38423, HIP 66290, HIP 107339) have listed $K_{s}$-band uncertainties in the 2MASS PSC of $>9$ mag. The documentation for the PSC (Cutri et al. 2003) indicates that these should not be interpreted as actual uncertainties, but as flags to indicate that the pipeline was unable to accurately estimate the uncertainty. Because good estimates of the uncertainty are critical (see below) for our determination of an IR excess, we now address better values for these errors.

HIP 38423 is also very bright, with $K_{s}<4$. It has $J$ and $H$ errors of $\sim 0.3 \mathrm{mag}$. Because we redetermined a $K$ magnitude for it above, it has a $K$ error of $0.06 \mathrm{mag}$. We do not identify an IR excess in this object.

The other three sources are not particularly bright; HIP 9782 has $K_{s}=6.74$, HIP 66290 has $K_{s}=5.63$, and HIP 107339 has $K_{s}=5.56$. The $J$ and $H$ uncertainties for all three of these stars are "normal," with values of order $0.02 \mathrm{mag}$. Using standard color-color relations, we combined estimates of $J-K_{s}$ and $H-K_{s}$ (based on the $B-V$ color) with the measured $J$ and $H$ magnitudes to predict a $K_{s}$. The predicted $K_{s}$ magnitudes are quite close to the observed values, leading us to conclude that the published values are reliable, despite the unusual uncertainty flags. We take the errors on the $K_{s}$ mag for these three stars as being 0.05 mag. None of these three objects appear to have IR excesses.

We note for completeness here that one source, HIP 61174 , also known as $\eta \mathrm{CrV}$, has relatively large 2MASS errors in all three bands; errors are reported as $\sim 0.2-0.3$ mag. This 
source is known to have a disk. Since it has a bright $K_{s}$ mag above, it has an adjusted $K_{s}$ and thus has adopted $K_{s}$ errors of $0.15 \mathrm{mag}$.

\subsubsection{WISE Data}

As mentioned above, we can use the WISE all-sky catalog to complement our sample. We extracted WISE measurements from the WISE catalog for each of our targets, matched by position. We took the PSF-fitted magnitudes and errors as reported by the catalog. It is important to note that the $3.4 \mu \mathrm{m}$ (W1) detections for the entire sample are saturated in the original data, and the values used here are obtained via PSF fitting to the wings of the detection. The $22 \mu \mathrm{m}$ (W4) points are, in contrast, not saturated. (The WISE Explanatory Supplement states that the channels saturate at 8.1, 6.7, 3.8, and -0.4 mag for $\mathrm{W} 1$, $\mathrm{W} 2, \mathrm{~W} 3$, and $\mathrm{W} 4$, respectively, but that fits to the unsaturated wings of the PSF allow viable magnitudes to be obtained up to $2.0,1.5,-3.0$, and -4 mag for the four channels, respectively.) For the majority of our sample, 255 stars, we have both [24] and [22] measurements. The $22 \mu \mathrm{m}$ points are generally offset from the $24 \mu \mathrm{m}$ points; see the Appendix for more details.

Out of our original sample defined above, there were a handful of objects that we removed from our proposal because we erroneously thought that there were Spitzer observations in the archive. We now thus include 10 WISE-only objects in our sample (see Table 1). We consider the implications of including these objects in our sample below. The blue-rapid sample has one WISE-only object, blue-slow has one that we ultimately dropped from the final sample (HIP 55897), red-rapid has six $W I S E$-only objects, and red-slow has two.

For each of the WISE-only objects, we investigated the images at each WISE band. Most of them appeared to be relatively clean point sources. HIP 32702 (in the red-rapid sample) seems to have somewhat unusual structure in the $22 \mu \mathrm{m}$ PSF, and has [3.4] $-[22]=-0.13$; we do not identify it as having an IR excess, and have left it in the sample. HIP 55897 (which would have been part of the blue-slow sample) nominally has a [3.4]-[22] $=0.29$, but by inspection of the W4 image, we consider it unlikely to have a real excess. We include it in Table 1 for completeness, but drop it from the rest of the analysis.

There is one object that seems, by comparison to the $K_{s}-[24]$ values as well as comparisons within WISE (e.g., [3.4]-[12]) to have either the 3.4 or $22 \mu \mathrm{m}$ measurement corrupted. HIP 3505 has $K_{s}-[24]=0$ and [3.4] $-[22]=0.90$. We suspect that this object does not really have a disk, and regard its WISE data as unreliable.

\subsubsection{Other IR Bands}

Six of the stars considered here, such as HIP $61174=\eta$ $\mathrm{CrV}$ and HIP $76829=\mathrm{HD}$ 139664, are already identified in the literature as having IR excesses due to a circumstellar disk, though often using (or including) wavelengths longer than $24 \mu \mathrm{m}$. For the purposes of our discussion here, we did not include information from wavelengths longer than $24 \mu \mathrm{m}$. We also did not include information, if available, from 4 to $20 \mu \mathrm{m}$. For the purposes of our discussion here, we wished to not introduce biases by including information for some objects (likely preferentially those with disks) obtained at other bands. We establish the presence or absence of an IR excess below using 22-24 $\mu \mathrm{m}$ alone. (See Table 3 for a complete list of literature disks identified using any wavelength.)

\subsubsection{Final Sample Sizes}

In the end, there are 263 stars in the final sample, having dropped 2 above, 1 with MIPS data and 1 WISE-only object. There are 49 stars in the blue-slow sample, 59 stars in the bluerapid sample, 78 stars in the red-slow sample, and 77 stars in the red-rapid sample. We present new MIPS measurements for 188 objects, measurements from MIPS data already in the archive for 67 objects ( 1 of which we dropped out of the sample), and WISE-only data for 10 objects (1 of which we dropped out of the sample).

\section{IDENTIFICATION OF INFRARED EXCESS OBJECTS}

\subsection{Selecting the Objects with Excesses}

In order to identify those objects that have an apparent IR excess and thus those that we will infer to have a circumstellar disk, we want to compare shorter-wavelength and longerwavelength bands. We need a band that samples the photosphere and a band that samples where the debris disk is expected to be bright. Preferably the two bands should be both on the Rayleigh-Jeans (RJ) side of the spectral energy distribution (SED) for these F stars, and be measurements that are as uniform (homogeneous) and well calibrated as possible, such that the scatter in the measurements (e.g., for those objects without disks) is minimized.

We have $V$ magnitudes for all of the stars in our sample, and the $V$ magnitude is likely to be sampling the photosphere in these stars. However, the $V$ measurements come from a variety of sources (so there is a lot of scatter in these values introduced by their various different origins), and the $V$ band for this sample of F stars is near the peak of the SED, so any color involving $V$ (e.g., $V-K_{s}$ or $V-$ [24]) will not have an expectation value of zero.

We have $K_{s}$ for all of the stars in our sample from 2MASS, and as such there is minimal scatter introduced from variations in calibration, data reduction systematics, etc. (except for the brightest stars, as discussed above). For these stars, which are not likely to have substantial inner disks, $K_{s}$ is likely to be sampling the photosphere. We checked the validity of all the $K_{s}$ magnitudes as discussed above. We examined the $J H K_{s}$ color-color diagram for our sample for any evidence of excesses at $K_{s}$ and found none. Moreover, $K_{s}$ is on the RJ side of the SED, such that, e.g., $K_{s}-$ [24] is expected to be zero and not vary over the (relatively small) range of types included here.

We have $24 \mu \mathrm{m}$ measurements for $97 \%$ of our sample. These data have all been re-reduced here so as to minimize data reduction systematics. MIPS data are known to be sensitive and have a stable PSF, such that the internal statistical error for the photometry is quite low. However the PSF, at 6", can include background (or foreground) objects close to the target. We selected our sample so as to be in relatively uncrowded fields so as to minimize this; see also the discussion below about contamination.

We also have WISE measurements at 3.4 and $22 \mu \mathrm{m}$ for all of our sample. These data are all uniform and internally consistently calibrated. The $3.4 \mu \mathrm{m}$ band, while longer than the $2.2 \mu \mathrm{m} K_{s}$ band, is likely to still sample photosphere, and the $22 \mu \mathrm{m}$ band should sample dust at a comparable distance from the star as the $24 \mu \mathrm{m}$ band. The spatial resolution (at $12^{\prime \prime}$ ) is poorer for WISE (than for MIPS), but as these fields are relatively uncrowded, this should have minimal impact. The data were obtained for the same objects nearly simultaneously, which minimizes any intrinsic variations in these stars at these bands. 
Table 3

Stars with IR Excesses or Needing Other Special Notes

\begin{tabular}{|c|c|c|}
\hline Name & Has IR Excess Here? & Notes \\
\hline HIP 1427 & Yes & $\chi_{24}>3, \chi_{22}<3$ but $\chi_{\text {best }}>3 ;$ new IR excess identified here \\
\hline HIP 3505 & No & One of the WISE bands is evidently wrong, so taking $\chi_{\text {best }}=\chi_{24}$ \\
\hline HIP 4868 & No & Binary \\
\hline HIP 5141 & No & Binary \\
\hline HIP 5144 & No & Binary \\
\hline HIP 5631 & Yes & $\chi_{24}>3, \chi_{22}<3$ but $\chi_{\text {best }}>3$; new IR excess identified here \\
\hline HIP 7916 & No & Binary \\
\hline HIP 8514 & No & Binary \\
\hline HIP 8805 & No & Binary; $K_{s}$ likely wrong, so taking $\chi_{\text {best }}=\chi_{22}$ \\
\hline HIP 9727 & No & Binary; aperture photometry used at $24 \mu \mathrm{m}$ \\
\hline HIP 9782 & No & Corrected large $K_{S}$ error in 2MASS \\
\hline HIP 10079 & No & Binary \\
\hline HIP 11191 & Yes & $\chi_{24}>3, \chi_{22}<3$ but $\chi_{\text {best }}>3$; new IR excess identified here \\
\hline HIP 12189 & No & Binary; = HD 16246, known planet host (Guenther et al. 2009) \\
\hline HIP 14235 & No & Binary \\
\hline HIP 16245 & No & Corrected $K_{s}, K_{s}$ error from bright 2 MASS $K_{s}$; binary \\
\hline HIP 16712 & No & Binary \\
\hline HIP 18187 & Yes & New IR excess identified here \\
\hline HIP 18735 & No & Binary \\
\hline HIP 18859 & Yes & $=\mathrm{HD} 25457$, known disk (Rhee et al. 2007); identified as having an IR excess here \\
\hline HIP 20693 & Yes & New IR excess identified here \\
\hline HIP 21066 & No & No [24] so $\chi_{\text {best }}=\chi_{22}$ \\
\hline HIP 21770 & No & Corrected $K_{s}, K_{s}$ error from bright 2 MASS $K_{s}$; binary \\
\hline HIP 22449 & No & Dropped from final sample as too bright at $K_{s}$ \\
\hline HIP 23395 & No & Binary \\
\hline HIP 23783 & No & Binary \\
\hline HIP 24947 & Yes & $\begin{array}{l}=\text { AS Col, known disk (Zuckerman et al. 2011); identified as having an IR excess } \\
\text { here }\end{array}$ \\
\hline HIP 25183 & Yes & Binary; new IR excess identified here \\
\hline HIP 26404 & No & Binary \\
\hline HIP 27633 & No & Binary \\
\hline HIP 28103 & No & $\begin{array}{l}\text { Corrected } K_{s}, K_{s} \text { error from bright } 2 \text { MASS } K_{s} ;=\eta \text { Lep }=\text { HD 40136, known disk } \\
\text { (Rhee et al. 2007) }\end{array}$ \\
\hline HIP 28498 & No & No $[24]$ so $\chi_{\text {best }}=\chi_{22}$ \\
\hline HIP 29650 & No & Binary \\
\hline HIP 31552 & Yes & $\chi_{24}>3, \chi_{22}<3$ but $\chi_{\text {best }}>3$; new IR excess identified here \\
\hline HIP 32644 & No & No $[24]$ so $\chi_{\text {best }}=\chi_{22}$ \\
\hline HIP 32702 & No & No [24] so $\chi_{\text {best }}=\chi_{22} ;[22]$ PSF may be suspect in image \\
\hline HIP 32851 & No & Binary \\
\hline HIP 33202 & No & Corrected $K_{s}, K_{s}$ error from bright 2 MASS $K_{s}$ \\
\hline HIP 34834 & No & Corrected $K_{s}, K_{s}$ error from bright 2MASS $K_{s}$ \\
\hline HIP 36165 & No & Binary \\
\hline HIP 36366 & No & Corrected $K_{s}, K_{s}$ error from bright 2 MASS $K_{s}$; binary \\
\hline HIP 36439 & No & Binary \\
\hline HIP 36485 & No & Binary \\
\hline HIP 36509 & No & No [24] so $\chi_{\text {best }}=\chi_{22}$ \\
\hline HIP 38423 & No & Corrected $K_{s}, K_{s}$ error from bright 2 MASS $K_{s}$; binary \\
\hline HIP 40843 & No & Corrected $K_{s}, K_{s}$ error from bright 2 MASS $K_{s}$ \\
\hline HIP 42163 & No & No [24] so $\chi_{\text {best }}=\chi_{22}$ \\
\hline HIP 42215 & No & No $[24]$ so $\chi_{\text {best }}=\chi_{22}$ \\
\hline HIP 43625 & No & $=$ HD 75616, known long-wavelength disk (Trilling et al. 2008) \\
\hline HIP 46535 & No & Binary \\
\hline HIP 47198 & Yes & $\chi_{24}<3, \chi_{22}<3$ but $\chi_{\text {best }}>3$; new IR excess identified here \\
\hline HIP 49809 & Yes & $\begin{array}{l}\chi_{24}>3, \chi_{22}<3 \text { but } \chi_{\text {best }}>3 ;=\text { HD } 88215 \text {, known disk (Trilling et al. 2007); } \\
\text { identified as having an IR excess here }\end{array}$ \\
\hline HIP 50384 & No & $\begin{array}{l}\text { Binary; }=\mathrm{HD} 89125, \text { listed in Koerner et al. (2010) as a disk, but likely to have } \\
\text { measured off-source background } 70 \mu \mathrm{m} \text { source }\end{array}$ \\
\hline HIP 50954 & No & Corrected $K_{s}, K_{s}$ error from bright 2MASS $K_{s}$ \\
\hline HIP 55897 & No & Only WISE data, but not clear object really there in W4 image, drop! \\
\hline HIP 61174 & Yes & $\begin{array}{l}\text { Corrected } K_{s}, K_{s} \text { error from bright } 2 \text { MASS } K_{s} ;=\eta \text { CrV, known disk (Stencel \& } \\
\text { Backman 1991); identified as having an IR excess here }\end{array}$ \\
\hline HIP 61621 & Yes & Binary; $\chi_{24}>3, \chi_{22}<3$ but $\chi_{\text {best }}>3 ;$ new IR excess identified here \\
\hline HIP 63503 & No & Corrected $K_{s}, K_{s}$ error from bright 2MASS $K_{s}$; binary \\
\hline HIP 64241 & No & Corrected $K_{s}, K_{s}$ error from bright 2 MASS $K_{s}$ \\
\hline HIP 66121 & No & Binary \\
\hline HIP 66290 & No & Corrected large $K_{s}$ error in 2MASS \\
\hline
\end{tabular}


Table 3

(Continued)

\begin{tabular}{|c|c|c|}
\hline Name & Has IR Excess Here? & Notes \\
\hline HIP 67918 & No & Binary \\
\hline HIP 69751 & No & Binary \\
\hline HIP 69989 & No & Binary \\
\hline HIP 71682 & No & Binary \\
\hline HIP 72197 & Yes & $\chi_{24}>3, \chi_{22}<3$ but $\chi_{\text {best }}>3$; new IR excess identified here \\
\hline HIP 72603 & No & Binary \\
\hline HIP 72682 & Yes & $\chi_{24}>3, \chi_{22}<3$ but $\chi_{\text {best }}>3$; new IR excess identified here \\
\hline HIP 73996 & No & Corrected $K_{s}, K_{s}$ error from bright 2MASS $K_{s}$; binary \\
\hline HIP 76829 & No & $\begin{array}{l}\text { Corrected } K_{s}, K_{s} \text { error from bright } 2 \text { MASS } K_{s} ;=\text { HD 139664, known } \\
\text { long-wavelength debris disk (Kalas et al. 2006) }\end{array}$ \\
\hline HIP 80008 & No & Binary \\
\hline HIP 80179 & No & Corrected $K_{s}, K_{s}$ error from bright 2MASS $K_{s}$ \\
\hline HIP 82860 & No & Corrected $K_{s}, K_{s}$ error from bright 2MASS $K_{s}$ \\
\hline HIP 83174 & No & Binary \\
\hline HIP 84883 & No & Binary \\
\hline HIP 88399 & Yes & $\begin{array}{l}=\text { HD 164249, known disk (Moór et al. 2011; Rebull et al. 2008; Rhee et al. 2007); } \\
\text { identified as having an IR excess here }\end{array}$ \\
\hline HIP 88728 & No & Binary \\
\hline HIP 90459 & No & Binary \\
\hline HIP 90596 & No & Binary \\
\hline HIP 90954 & No & Binary \\
\hline HIP 91237 & No & Binary \\
\hline HIP 91421 & No & Binary \\
\hline HIP 92270 & Yes & $\chi_{24}>3, \chi_{22}<3$ but $\chi_{\text {best }}>3$; new IR excess identified here \\
\hline HIP 96441 & No & Corrected $K_{s}, K_{s}$ error from bright 2 MASS $K_{s}$; binary \\
\hline HIP 97016 & No & Binary \\
\hline HIP 97650 & No & Binary \\
\hline HIP 99273 & Yes & $\begin{array}{l}=\text { HD 191089, known disk (Rhee et al. 2007); identified as having an IR excess } \\
\text { here }\end{array}$ \\
\hline HIP 99352 & No & Binary \\
\hline HIP 99572 & No & Binary \\
\hline HIP 99680 & No & PRF fitting reveals two sources within $\sim 5^{\prime \prime} .5$, so taking $\chi_{\text {best }}=\chi_{24}$ \\
\hline HIP 100800 & Yes & New IR excess identified here \\
\hline HIP 103389 & No & $\begin{array}{l}=\text { HD 199260, known long-wavelength disk (Beichman et al. 2006; Lawler et al. } \\
\text { 2009) }\end{array}$ \\
\hline HIP 103635 & No & Binary \\
\hline HIP 105202 & No & Binary \\
\hline HIP 105547 & No & Binary; PRF fitting finds two sources within $\sim 5^{\prime \prime} .5$, so taking $\chi_{\text {best }}=\chi_{24}$ \\
\hline HIP 106395 & No & Binary \\
\hline HIP 107300 & No & Binary \\
\hline HIP 107339 & No & Corrected large $K_{s}$ error in 2MASS; no [24] so $\chi_{\text {best }}=\chi_{22}$ \\
\hline HIP 107947 & Yes & $\begin{array}{l}\chi_{24}>3, \chi_{22}<3 \text { but } \chi_{\text {best }}>3 \text {; }=\text { HD } 207575 \text {, possible disk (Zuckerman et al. } \\
\text { 2011); identified as having an IR excess here }\end{array}$ \\
\hline HIP 108809 & Yes & $\begin{array}{l}\chi_{24}>3, \chi_{22}<3 \text { but } \chi_{\text {best }}>3 ;=\text { HD } 209253 \text {, known disk (Rhee et al. 2007); } \\
\text { identified as having an IR excess here }\end{array}$ \\
\hline HIP 113502 & No & No $[24]$ so $\chi_{\text {best }}=\chi_{22}$ \\
\hline HIP 114270 & No & Binary \\
\hline HIP 114665 & No & Binary \\
\hline HIP 114948 & Yes & $\begin{array}{l}\chi_{24}>3, \chi_{22}<3 \text { but } \chi_{\text {best }}>3 ;=\text { HD } 219482 \text {, known disk (Beichman et al. } \\
\text { 2006); identified as having an IR excess here }\end{array}$ \\
\hline
\end{tabular}

So, which indicator should we use for identification of IR excess sources? As discussed in the Appendix, the intermingling of $K_{s}$, [24], and WISE is somewhat problematic. However, $K_{s}-[24]$ and [3.4]-[22] are two entirely independent measures of IR excess. Figures 2 and 3 show the $K_{s}$ versus $K_{s}-[24]$ and [3.4] versus [3.4]-[22] plots for the sample, respectively. As can be seen in the plots, these distributions are similar in that the bulk of the distribution is scattered about 0 and there are outliers to the red (right) that we infer to be the disk candidates. The extremely red objects in Figures 2 and 3 are the same objects. In Figure 2, the distribution is relatively tightly distributed about the $K_{s}-[24]=0$ line, though it is slightly offset to the blue such that the most likely value in the distribution (the mode of the distribution) is actually $K_{s}-[24]=-0.03-$ this is most likely primarily a result of some combination of our particular reduction of the MIPS data, plus uncertainties in absolute calibration between the $24 \mu \mathrm{m}$ and $K_{s}$ systems. The distribution develops more scatter at the brighter end, where the $K_{s}$ values are not as reliable (as per discussion above), and there are fewer of them. In Figure 3, [3.4]-[22] is zero for most of the ensemble, and the scatter about the [3.4]-[22] $=0$ distribution is somewhat larger than that for $K_{s}-[24]$, but better centered on 0 , and does not appear to disperse quite so obviously at the brighter end. 


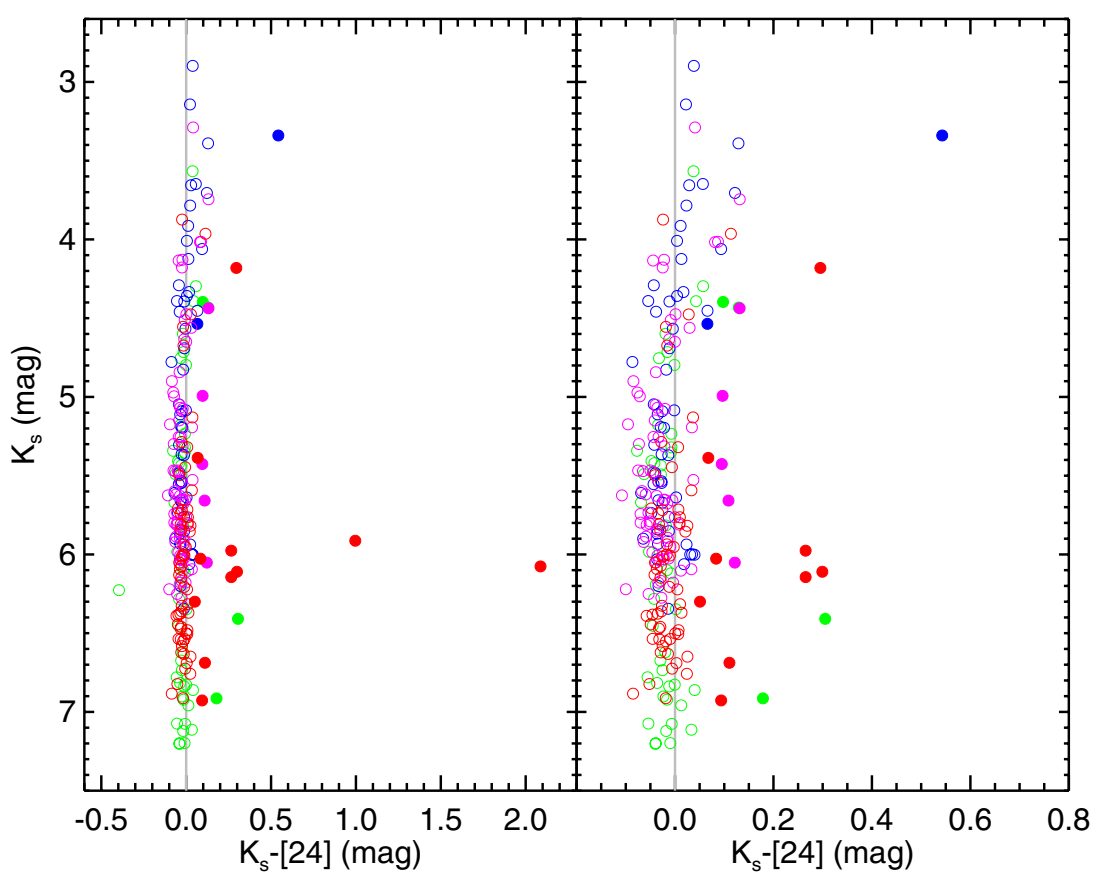

Figure 2. $K_{s}$ vs. $K_{s}-$ [24] for our sample. The light gray line is at $K_{s}-[24]=0$. The left panel is full scale; the right panel zooms in on the $K_{s}-[24]=0$ regime. Open circles mark the non-excess sample and closed circles mark the selected infrared excesses as described in the text. Green circles represent the blue-slow group, blue circles represent the blue-rapid groups, magenta circles represent the red-slow group, and red circles represent the red-rapid group. Note that the bulk of the distribution is not centered on 0 ; see the text. The object with a large negative excursion is also discussed in the text.

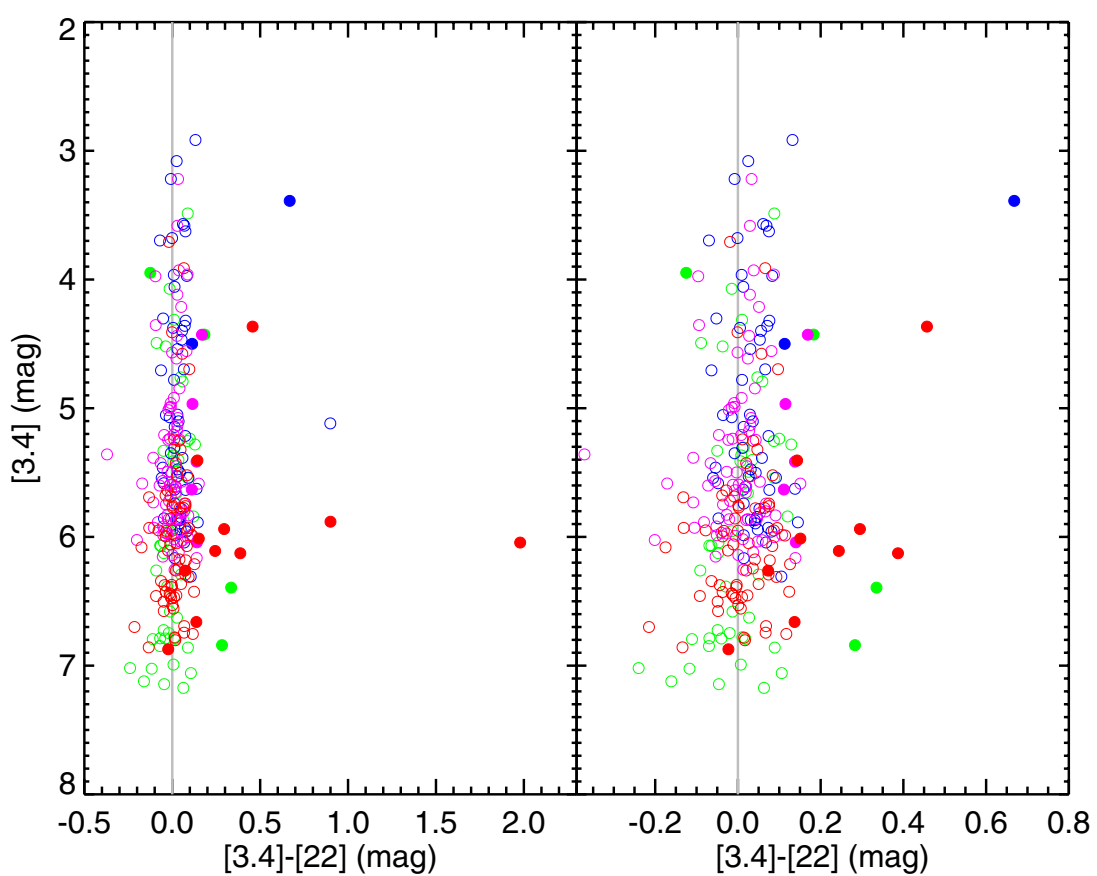

Figure 3. [3.4] vs. [3.4] - [22] for our sample. The light gray line is at [3.4] $-[22]=0$. The left panel is full scale; the right panel zooms in on the [3.4] $-[22]=0$ regime. Notation is as in the previous figure, where open circles mark the non-excess sample and closed circles mark the selected infrared excesses as described in the text. The object selected as an IR excess with a large negative excursion here (HIP 99680) and the object with [3.4]-[22] $>0.5$ that is not selected as having an IR excess (HIP 3505) are identified as having bad WISE measurements, and so are selected based on their $K_{s}-$ [24] values; see the text.

We investigated seven options for determining where to set the limit between disk candidate and photosphere candidate at 22 or $24 \mu \mathrm{m}$. We now discuss each of these in turn; the last option is the one we selected.

1. We looked at simply setting a limit of $+0.1 \mathrm{mag}$ in the $K_{s}-[24]$ color or $+0.2 \mathrm{mag}$ in the [3.4]-[22] color; there is a gap at $[3.4]-[22]=0.2$ that is tempting to use. However, there is no such gap in the $K_{s}-$ [24] distribution, and in theory, we are more sensitive to weaker disks using [24] rather than [22].

2. We tested a method by Sierchio et al. (2010), which for a given star uses its $B-V$ color to predict its $H-K$ color from the Kenyon \& Hartmann (1995) table. From the observed $H$ and predicted $H-K$ color, an estimated $K$ magnitude was calculated. This estimated $K$ magnitude was then averaged 
with the observed $K_{s}$ magnitude to produce what Sierchio et al. (2010) found to be a better estimate of $K$ for their sample. This method, for our data sample, did not give a significantly lower scatter in our observed data, so we used the observed $K_{s}$ magnitudes for this study, except where noted in Section 2.4.

3. We tested a method suggested by Urban et al. (2012) which uses $V-K_{s}$ to predict the $K_{s}-$ [24] values expected, but for the narrow range of $\mathrm{F}$ stars considered here, this is equivalent to assuming $K_{s}-$ [24] should be 0 (or indistinguishably close to it), so it yields identical results to one of the other methods considered here.

4. We empirically assessed the scatter in the $K_{s}-[24]$ and [3.4]-[22] distributions as a function of brightness via computing a running mean and standard deviation $(\sigma)$, and attempted to set a $3 \sigma$ limit based on that scatter. The scatter in our $K_{s}-$ [24] data is consistent with (or better than) other similar studies in the literature. The scatter indicates the overall empirical uncertainty in the calculated $K_{s}-$ [24] and [3.4]-[22] distributions, and gets larger at fainter magnitudes; however, it does not take into account the uncertainties on the individual measurements, and it becomes more difficult to calculate the scatter at the bright end, due to decreasing numbers of objects.

5 . and 6 . We incorporated the errors on the measurements by calculating

$$
\chi_{24}=\frac{\left(K_{s}-[24]\right)_{\mathrm{observed}}-\left(K_{s}-[24]\right)_{\text {predicted }}}{\sigma_{\left(K_{s}-[24]\right)}}
$$

as per, e.g., Trilling et al. (2008). For $\chi_{24}$, the expected (predicted) $K_{s}-[24]$ for our data reduction is evidently slightly blue at -0.03 , since that is the most likely value found in the distribution of $K_{s}-$ [24] values. As a completely different assessment (which we take to be disk option 6), we similarly calculated $\chi_{22}$; we took the expected value of [3.4]-[22] to be 0 . The limit for significant IR excess is usually taken to be $\chi_{x}=3$. When selecting IR excess objects using just $\chi_{24}>3,21$ objects are selected; using just $\chi_{22}, 11$ objects are selected. These objects are not quite the same between the two selections, due to uncertainties in the individual points, e.g., cases like HIP 8805 discussed above, where $K_{s}$ is likely wrong. There are nine objects for which both $\chi_{24}$ and $\chi_{22}$ are $>3$ : HIP 18187, HIP 18859, HIP 20693, HIP 24947, HIP 25183, HIP 61174, HIP 88399 HIP 99273, and HIP 100800. There are, however, many objects with "borderline" $\chi$ values, more so for $\chi_{24}$ than $\chi_{22}$-for these objects, $\chi_{24}$ is near but not quite 3 (more on these objects below).

Essentially all of these methods select the same seven stars with the most extreme values of $K_{s}-$ [24] and [3.4]-[22], and these are likely the most secure disk candidates (HIP 18187, HIP 18859, HIP 20693, HIP 25183, HIP 61174, HIP 88399, HIP 99273). These objects all have $K_{s}-[24] \geqslant 0.265$ and [3.4] $-[22] \geqslant 0.295$ - though we note that there is one more IR excess object (HIP 24947) with $K_{s}-[24]=0.265$ but [3.4] - [22] $=0.244$, so not quite as extreme in [3.4] - [22], though clearly identified as having an IR excess.

There are many objects selected by only one or two methods out of the several we tested. There are some objects that have obviously incorrect values in one color but not the other, rendering methods using only the "wrong" color less

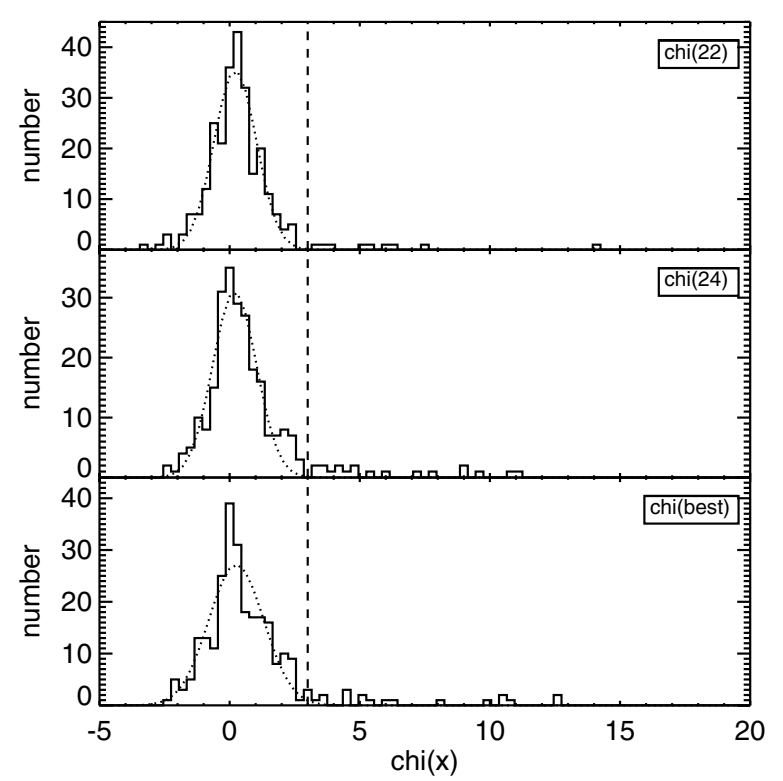

Figure 4. Histograms of $\chi_{22}$ (top), $\chi_{24}$ (middle), and $\chi_{\text {best }}$ (bottom) for the ensemble. Dotted lines are Gaussian fits to the distributions. Note that extreme negative and extreme positive values of $\chi$ are not shown here.

than optimal. For example, HIP 8805, discussed above as likely having an incorrect $K_{s}$, has $K_{s}-[24]=-0.40$ but [3.4]-[22] $=0.05$, suggesting that indeed, its $K_{s}$ is incorrect, and that it does not have a detectable IR excess. It can also happen that one or more of the WISE bands are likely incorrect; as discussed above, HIP 99680 has [3.4]-[22] $=-0.37$, the bluest of the ensemble, and $K_{s}-[24]=0.14$, so it is likely that either [3.4] or [22] is not correct for this object. However, the overwhelming majority of the sample has $K_{s}-$ [24] and [3.4]-[22] measurements that are consistent with each other (and therefore $\chi_{24}$ and $\chi_{22}$ values that are consistent with each other). There are objects that show up as marginally significant in both bands independently, e.g., $\chi_{22} \sim \chi_{24} \sim 2.8$.

We examined the distributions of $\chi_{22}$ and $\chi_{24}$; see Figure 4. The histograms are generally well behaved, in that there is a strong peak near 0 for both. The outliers on the blue $\left(\chi_{x}<0\right)$ side can, in both cases, be attributed (as for HIP 8805 or HIP 99680) to bad values in one of the relevant bands. Both distributions have a long tail to the red $(\chi>0)$, as expected. The histogram for $\chi_{22}$ is nicely Gaussian, and $\chi_{22}=3$ seems visually to be the right cutoff given the distribution; there is a gap near $\chi_{22} \sim 3$ corresponding essentially to the gap seen near [3.4]-[22] $=0.2$. The bestfit Gaussian to this distribution suggests that a $3 \sigma$ cutoff would be $\chi_{22} \sim 2.4$ rather than 3 . The histogram for $\chi_{24}$ is not quite as clearly Gaussian, with a more prominent asymmetry to the red. While there is also a visible gap near $\chi_{24} \sim 3$, and a Gaussian fit to the distribution suggests that a $3 \sigma$ cutoff would be $\chi_{24} \sim 2.5$ rather than 3 , the $\chi_{24}$ distribution deviates from a Gaussian with a red "bump" near $\chi_{24}=2$ such that there are $\sim 15$ objects right at that boundary.

7. We would like to take into account information from all four bands $\left(K_{s}\right.$, [3.4], [22], and [24]), compensating for missing or obviously incorrect values, and make a viable decision on what objects should or should not be disk candidates. Since $K_{s}-$ [24] and [3.4]-[22] are two entirely different and independent measures of IR excess, we wished to combine 
them. We calculated $\chi_{22}$ and $\chi_{24}$ for each and then took all objects for which

$$
\chi_{\text {best }}=\frac{\chi_{22} \sigma_{\left(K_{s}-[24]\right)}+\chi_{24} \sigma_{([3.4]-[22])}}{\sqrt{\sigma_{([3.4]-[22])}^{2}+\sigma_{\left(K_{s}-[24]\right)}^{2}}} \geqslant 3
$$

as IR excess candidates. In some cases, where data are missing or obviously incorrect, the $\chi_{\text {best }}$ was taken to be either $\chi_{22}$ or $\chi_{24}$, as appropriate, and these are noted in Table 3 . This merging of the two different values of $\chi$ (where available) ameliorates inaccurate measurements in any of the four bands (particularly those for which, e.g., one band has a large uncertainty), takes advantage of all the information we have, and incorporates the idea that the significance of the excess should be inexorably tied to the size of the uncertainty on the measurement. It includes as disks those things that are borderline in both (e.g., $\chi=2.5$ in both independent measures, or 2.97 in one and 2.5 in the other), and does not include those things that are borderline in one but not the other (e.g., 2.97 in one and 0.8 in the other). There are 13 objects in our final IR excess selection with $\chi_{22}<3$. There is one object with $\chi_{24}<3$, and it also happens to have $\chi_{22}<3$ (HIP 47198).

The distribution of $\chi_{\text {best }}$ is shown in Figure 4, and it is the least Gaussian of the three distributions in that figure. The definition of $\chi_{\text {best }}$ is consistent with this effect. The best-fit Gaussian is consistent with a cutoff of $\chi_{\text {best }}=3$ for a significant excess.

All values of $\chi$ are provided in Table 1 so that readers can make their own judgments as to the strength and reliability of the excess. Note that $K_{s}$, [24], [3.4], [22], $K_{s}-$ [24], and [3.4]- [22] are given (with errors) to $0.001 \mathrm{mag}$-more than is necessarily warranted given the precision of the results, but this aids in reproduction of our values of $\chi$. We are taking $\chi_{\text {best }} \geqslant 3$ as our IR excess candidates for the remainder of this paper. The objects indicated as having an IR excess in all the figures here are those selected via this mechanism.

\subsection{Limits on Contamination}

Empirically, within the $\sim 300$ objects we examined in the course of this work, one object (HIP 32435) was discarded as confused with a background galaxy. We now consider those cases in which we would not be able to resolve and thus identify a contaminant, whether a background galaxy or an asteroid.

As in Stauffer et al. (2005), we determined the expected rate at which background active galaxies projected onto the line of sight of our target stars would produce false-positive apparent infrared excesses at $24 \mu \mathrm{m}$. We used the $24 \mu \mathrm{m}$ source counts in Papovich et al. (2004) to calculate the probability of a chance superposition of a ULIRG and of our target stars. Because the contamination rate increases toward lower flux levels, as a conservative measure we only calculated the contamination rate for our faintest sources. There are 98 stars in our sample with $K_{s}>6$. Out of those 98, 92 have a $24 \mu \mathrm{m}$ flux measurement; their mean $24 \mu \mathrm{m}$ flux density is $\sim 22 \mathrm{mJy}$. Our smallest excess limit of $\sim 8 \%$ then implies that a contaminating ULIRG would need a flux density greater than $\sim 1.8 \mathrm{mJy}$ to cause us to misidentify a bare photosphere as an IR excess object. Using data from Figure 2 of Papovich et al. (2004), there are $\sim 10^{6}$ objects $\mathrm{sr}^{-1}$ or $\sim 2.3 \times 10^{-5}$ objects $\operatorname{arcsec}^{-2}$ with $24 \mu \mathrm{m}$ flux density greater than this. Combining this estimate with our $13^{\prime \prime}$ aperture radius yields a predicted contamination rate of 0.012 for each of our faint target stars, or a $1.2 \%$ chance that one of our faint stars will have a false excess detection due to ULIRG contamination. This rate will be even lower since we are using PSF-fitting photometry, not aperture photometry, for most of our MIPS sources. WISE data, though they have a larger PSF, are also PSF-fitted photometry, and are overall less sensitive than MIPS; the net rate of contamination in the WISE data is likely to be comparable.

Asteroids are not a concern for this project. Taking the Taurus Spitzer Survey (D. D. Padgett et al. 2012, in preparation; Rebull et al. 2010) as a worst-case scenario because the survey is taken in the ecliptic plane, we would expect 0.0032 asteroids on a given aperture, even in the ecliptic plane. When taken 300 times, to approximate our sample, we expect to find an asteroid contaminating our target $0.96 \%$ of the time. Since our targets are all over the sky and not all in the ecliptic plane, the real contamination rate is even lower. While the WISE PSF is larger, the data acquisition scheme for WISE is such that moving targets can be removed, and so it is very unlikely that anything remaining in the WISE catalog are asteroids.

To investigate the chances of randomly finding stars with excesses (due to real or contaminated sources), we conducted Monte Carlo experiments. For groups of 50 stars (to approximate each of our color/rotation groups), we drew a random set of stars from a master pot of simulated stars with a known disk fraction. For a "true" disk fraction of $5 \%$, there is a $<10 \%$ chance of finding five or more disks, as for the red-rapid sample. For a "true" disk fraction of $2.5 \%$, there is a $<0.6 \%$ chance of finding five or more disks. This is consistent with expectations from binomial statistics (11\% chance of five or more disks for $P=0.05$ and $0.8 \%$ chance for five or more disks for $P=0.025)$.

\subsection{WISE Additions}

We have added to our sample some objects with only WISE data and no MIPS data. In order to investigate whether this might be implicitly biasing our results, we looked more closely at the distributions of $\chi_{22}$ and $\chi_{24}$.

For the majority of our sample, $\chi_{22}$ and $\chi_{24}$ are consistent with each other. If there is a large $K_{s}-[24]$, then there is also a large [3.4]-[22] and vice versa. In those borderline cases where the $\chi$ from either $K_{s}-[24]$ or [3.4]-[22] is near 3 but less than 3 , the additional information obtained from the additional measurements can move the object over into the regime of believable IR excesses. For this sample, the distribution of $\chi_{22}$ seems to be somewhat better behaved in that the distribution is closer to a Gaussian. Out of the 13 objects that have $\chi_{22}<3$ and/or $\chi_{24}<3$ but $\chi_{\text {best }}>3$, all also have $\chi_{22}<\chi_{24}$. If we had only the WISE data for these stars, these borderline objects would not have been selected as IR excess objects. Among the sample of objects selected to have IR excesses, the lowest value of $\chi_{22}$ for any object with well-behaved [3.4] and [22] is $\sim 1.2$.

There are nine WISE-only sources in the final sample scattered fairly evenly through the distributions. None of the WISE-only sources have $\chi_{22}>3$. There are four sources out of those nine that have $\chi_{22}>1.2$, so these, if we had supporting MIPS data, might have small IR excesses, e.g., weak disks. This is likely within the errors we consider below.

\subsection{Binaries}

Our sample selection mitigated against equal-mass or nearequal-mass binaries, but many are in fact known (apparent) binaries, and our knowledge of this information may be incomplete. 


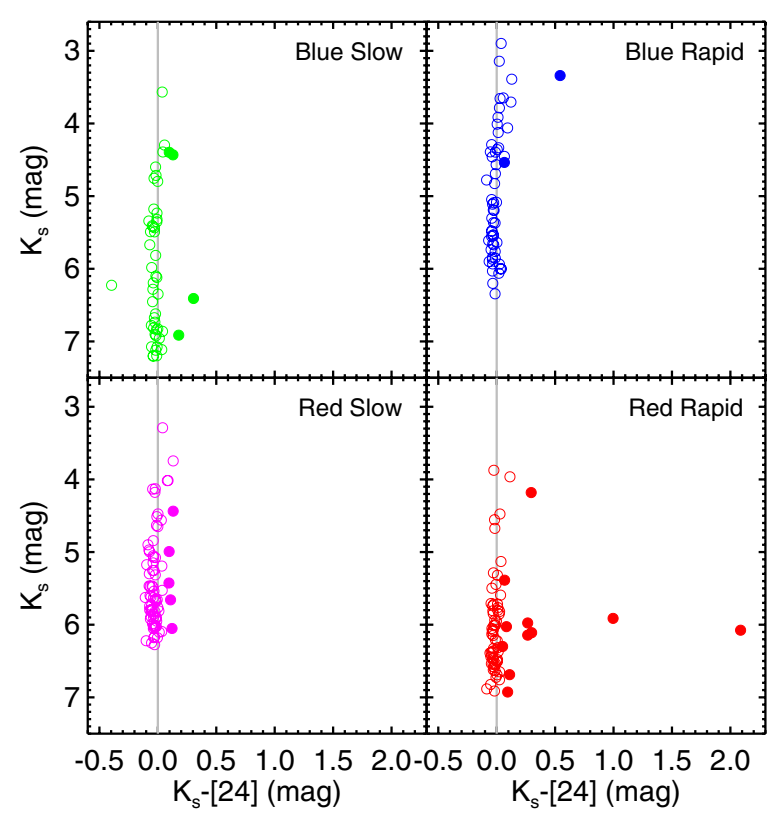

Figure 5. $K_{s}$ vs. $K_{s}-$ [24] plot of each group individually and their $24 \mu \mathrm{m}$ excesses; colors and symbols are as in the previous figure. The most excess stars are found in the red-rapid sample, but given small number statistics, this is not significantly different than the other samples. See Section 3 for discussion. (A color version of this figure is available in the online journal.)

Low-mass companions could make an apparent IR excess. A simple comparison of blackbody models at the appropriate $T_{\text {eff }}$ suggests that an M star companion to our F stars could be contributing between $\sim 25 \%$ and $35 \%$ of the flux at $24 \mu \mathrm{m}$. Among our candidates for IR excess, the distribution of fractional measured excesses (meaning (measured-predicted)/predicted) is broad, but there is a pileup of values at the $15 \%-20 \%$ level. If the more conservative criterion of $\chi_{24}>3$ is adopted, there is still a pileup of fractional excess at $24 \mu \mathrm{m}$ at the $15 \%-20 \%$ level; if one adopts $\chi_{22}>3$, the pileup occurs at $\sim 30 \%$.

However, if there is an M star companion, $K_{s}-[24]$ would not be zero for that photosphere (Gautier et al. 2007). Taking $K_{s}-[24] \sim 0.3$ as typical, if there is an unresolved M star that makes up $20 \%$ of the total flux, then the net photosphere might be $\sim 5 \%-10 \%$ brighter in $K_{s}-$ [24]. Moreover, this issue would only matter if there was an apparent binary with a separation of a few arcseconds such that $K_{s}$, the highest spatial resolution observation of the four bands, resolved a pair of stars, but MIPS did not. In this case, neither WISE band would be able to resolve the stars either, and the [3.4]-[22] would be less affected than the $K_{s}-$ [24]. It is unlikely that there are many cases like this in our sample.

When obtaining the measured $v \sin i$, some binaries were identified-59 objects $(23 \%)$ out of the entire set. These are indicated in Table 3. However, only two of them are identified as having an excess, HIP 25183 and HIP 61621. It does not appear that binaries are a significant problem for our data set.

\subsection{Net Disk (Excess) Frequency}

Having established our criteria for selecting significant IR excesses above, we find a net $\sim 8 \%$ excess rate; 22 out of 263 stars in our sample have significant infrared excesses at 22-24 $\mu \mathrm{m}$. Figures 2 and 3 identify those stars with excesses; Figure 5 plots $K_{s}$ versus $K_{s}-$ [24] for each sub-sample individually, with excesses marked.
Overall, three stars in our sample (HIP 61174, HIP 88399, HIP 99273) have $K_{s}-[24]>0.5$ (and [3.4] - [22] > 0.6), and are the most unambiguous disks. All three of them are rapid rotators, and previously identified disks (see Table 3). HIP 61174 is $\eta \mathrm{CrV}$, first noted as having a disk by Stencel \& Backman (1991), and is the brightest disk of the sample. HIP 88399 is HD 164249, part of the Beta Pic Moving Group, and identified by several authors as having a disk (Moór et al. 2011; Rebull et al. 2008; Rhee et al. 2007). HIP 99273, the largest $K_{s}-$ [24] and [3.4] - [22] in the sample, is HD 191089, identified as having a disk by Rhee et al. (2007).

Out of the next five stars in our sample with the next largest $K_{s}-$ [24] excesses (HIP 18187, HIP 18859, HIP 20693, HIP 24947, HIP 25183), two have previously been noted as having a disk. HIP 18859 is HD 2545 and was identified as a disk by Rhee et al. (2007). HIP 24947 is AS Col, which was identified as a disk by Zuckerman et al. (2011). The rest are newly identified excess stars. They have $K_{s}-$ [24] from 0.27-0.3, and [3.4] - [22] from 0.24 to 0.46 . Another star, HIP 100800 , is next in ordering by $24 \mu \mathrm{m}$ excess, with $K_{s}-[24]=0.18$ but [3.4] - [22] $=0.28$; this is another newly identified disk.

The remaining 13 stars we identify as having an IR excess (see Tables 1 and 3) are all more moderate excesses, with $K_{s}-$ [24] between 0.03 and $0.13 \mathrm{mag}$, and [3.4]-[22] between 0.07 and $0.18 \mathrm{mag}$-with two of the objects having possibly questionable [3.4]-[22] $<0$. Four of these lower $K_{s}-$ [24] objects are previously identified disks in the literature-HIP 108809 (Rhee et al. 2007), HIP 107947 (Zuckerman et al. 2011), HIP 49809 (Trilling et al. 2007), and HIP 114948 (Beichman et al. 2006). There is only one of these lower-excess objects in the blue-rapid sample; the rest are scattered among the other three sub-samples.

How does our overall excess frequency compare to previous determinations for similar target populations? Perhaps the best comparison is to the Formation and Evolution of Planetary Systems (FEPS) sample (Meyer et al. 2006; Carpenter et al. 2008, 2009). FEPS obtained Spitzer data using all the instruments for a sample of $314 \mathrm{~F}, \mathrm{G}$, and K dwarfs. Because the FEPS program was designed to determine how debris disks evolve with time, their sample was significantly weighted to young ages where debris disks are a priori more likely to be detectable; in particular about $20 \%$ of their stars are younger than $20 \mathrm{Myr}$, whereas few to none of our sample are likely to be that young. Our stars are expected to be field stars, and $\sim 60 \%$ of the FEPS sample is also field stars. The F stars make up only about $13 \%$ of the entire FEPS sample, and about $15 \%$ of the sample $>20$ Myr. Excluding the age $<20$ Myr stars from their sample (but retaining stars of all spectral types), and using their flux density ratio of $24-8 \mu \mathrm{m}$ as a proxy for the excess above photospheric flux, 21 of 232 of their stars have $24 \mu \mathrm{m}$ excesses exceeding $10 \%$ above the photosphere. This yields an excess (disk) frequency of about $9 \%$, slightly more than what we obtain $(8 \%)$. They note that the excess frequency drops at older ages, to $\sim 3 \%$ for age $>300$ Myr. Our sample of stars is more likely to be closer in age to $300 \mathrm{Myr}$ than theirs, on average. On the other hand, Meyer et al. (2008) note that the cluster stars show a higher-excess fraction (at $24 \mu \mathrm{m}$ ) than a comparable field sample of a given age; our stars are likely to be field stars. Our excess frequency of $\sim 8 \%$ is consistent with their measurement.

Another recent sample of F stars, Moór et al. (2011), finds 27 disks out of $82 \mathrm{~F}$ stars, or $33 \%$. However, as discussed in that paper, the sample was deliberately selected so as to be biased 


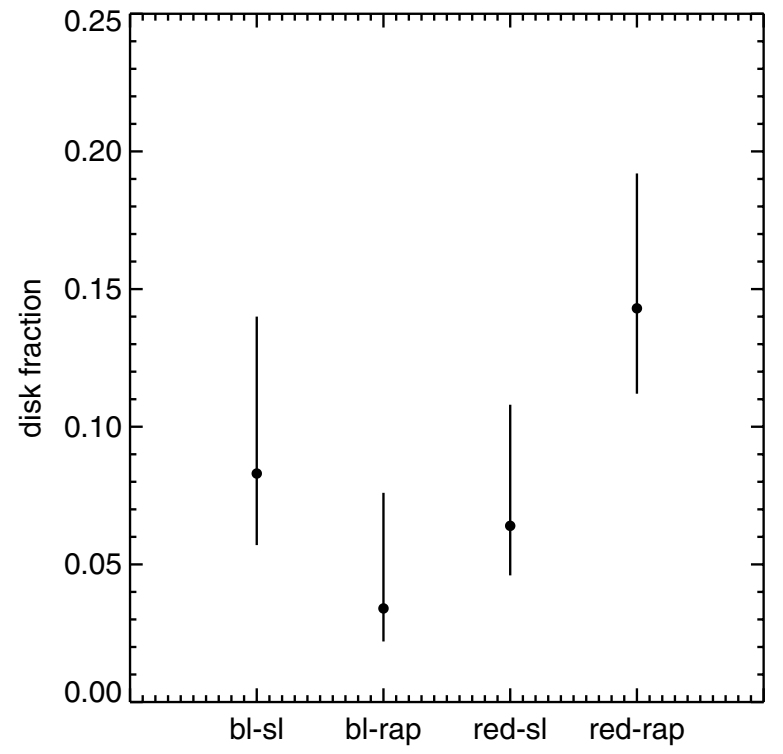

Figure 6. Plot of disk fractions as calculated in this paper, with formal errors calculated as per Burgasser et al. (2003). The $x$-axis is, from left to right, blueslow, blue-rapid, red-slow, and red-rapid. The red-rapid sample has the largest fraction of disks, and the blue-rapid sample has the smallest fraction of disks.

Table 4

Excess Results

\begin{tabular}{lcccc}
\hline \hline Group Name & No. of Stars & No. of Excesses & Excess Fraction & $1 \sigma$ Errors \\
\hline Blue-slow & 49 & 4 & 0.083 & $0.057-0.140$ \\
Blue-rapid & 59 & 2 & 0.034 & $0.022-0.076$ \\
Red-slow & 78 & 5 & 0.064 & $0.046-0.108$ \\
Red-rapid & 77 & 11 & 0.143 & $0.112-0.192$ \\
\hline
\end{tabular}

toward likely harboring a disk. Thus, that sample cannot be directly compared to our sample.

\section{DISCUSSION AND CONCLUSIONS}

The disk (excess) fractions are listed explicitly in Table 4. The blue-rapid sample has the lowest disk fraction at $\sim 3 \%$, and the red-slow has the next lowest disk fraction at $\sim 6 \%$. The blue-slow sample has a disk fraction of $\sim 8 \%$, and the red-rapid sample has the highest disk fraction at $\sim 14 \%$. The errors on these disk fractions can be calculated using the binomial distribution, as per Burgasser et al. (2003); Table 4 lists these formal errors, and Figure 6 represents that information graphically.

Among the entire sample, we find the highest fraction of IR excesses (and inferred disks) in the red-rapid rotators $(\sim 14 \%)$. There are $\sim 8 \%$ disks in the sample opposite in properties, the blue slowly rotating sample. The lowest disk fraction is the bluerapid sample $(\sim 3 \%)$, which is consistent with the blue-slow and red-slow samples and is perhaps distinct in disk fraction from the red-rapid sample $(\sim 14 \%)$. The red-rapid sample may have significantly more disks than the blue-rapid sample, but both blue samples are consistent with each other, and both red samples are (barely) consistent with each other. Both slow samples are consistent with each other, and the two rapid samples may be significantly different from each other.

Taken at face value, these disk fractions are not what we expected, a priori, to find. If winds clear disks, and if early $\mathrm{F}$ stars do not have winds, then we would have expected a high disk fraction for both of the blue samples (total of $6 / 108=$ $6 \%$, error range $4 \%-9 \%$ ) compared to the red samples (total of

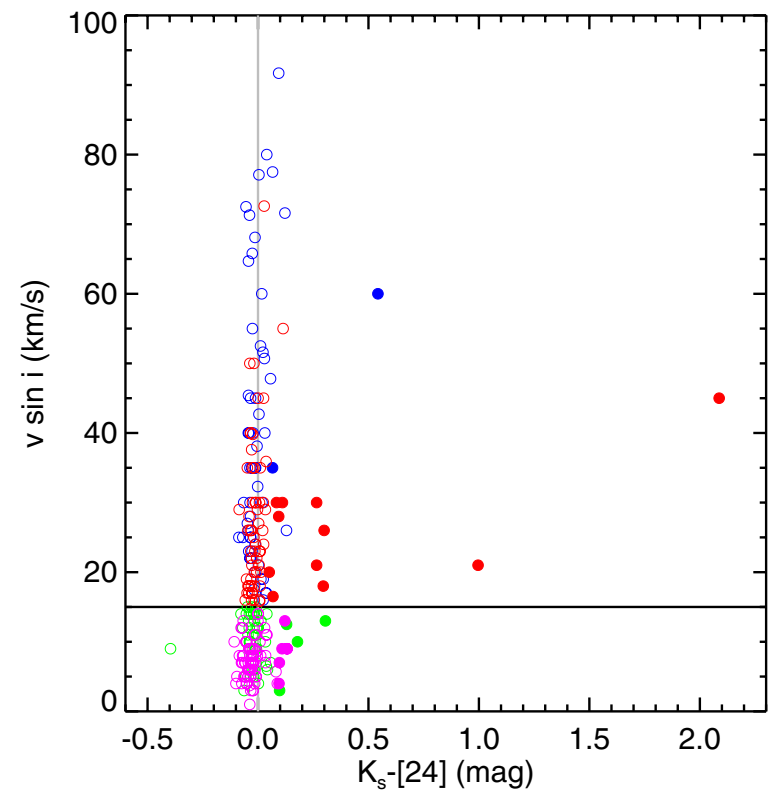

Figure 7. $v \sin i$ vs. $K_{s}-[24]$ plot for our sample; notation is as in previous figures. This figure shows the range of rotation rates; the division between slow and rapid rotators (indicated) is $v \sin i \sim 15 \mathrm{~km} \mathrm{~s}^{-1}$.

$16 / 155=10 \%$, error range $8 \%-13 \%$ ). We do not see that they are even consistent with each other, taking both extremes of the errors. The division between red and blue stars corresponding to a type of F5 is not particularly subject to debate. The nature of the winds on either side of this boundary almost certainly is different. However, we cannot discern any significant difference in the disk fractions between the red and blue samples.

The division we used between rapid and slow rotators is $15 \mathrm{~km} \mathrm{~s}^{-1}$. As discussed above, this was selected based on where the X-ray flux saturates for $\mathrm{G}$ stars, and the sub-samples were constructed to be approximately equally sized, given this cutoff (136 rapidly rotating objects, and 127 slowly rotating objects). Their overall disk fractions are $13 / 136=10 \%$ (error $8 \%-13 \%$ ) and $9 / 127=7 \%$ (error 5\%-10\%). Figure 7 shows a $v \sin i$ versus $K_{s}-$ [24] plot for our whole sample, with our rapid/slow division indicated in the plot. If one instead were to use a division of the order of $30 \mathrm{~km} \mathrm{~s}^{-1}$, however, this plot appears to show a low excess frequency for rapid rotators and a relatively high excess frequency for slow rotators. However, the correlation is illusory, in large part because of the distortion of the sub-sample sizes (53 objects with $v \sin i>30 \mathrm{~km} \mathrm{~s}^{-1}$ and 210 objects with $v \sin i \leqslant 30 \mathrm{~km} \mathrm{~s}^{-1}$ ). Nearly $65 \%$ of the 53 stars with $v \sin i>30 \mathrm{~km} \mathrm{~s}^{-1}$ are early $\mathrm{F}$ stars. Were we to use $30 \mathrm{~km} \mathrm{~s}^{-1}$ instead as the cutoff between rapid and slow, the disk fractions would be $2 / 34$ (6\%, blue-rapid), $1 / 19$ (5\%, redrapid), 4/74 (5\%, blue-slow), and 15/136 (11\%, red-slow). The sub-samples are so different in size that comparison of them is very difficult, and these disk fractions are identical to what we have already determined for these samples within small number statistics. We will retain our rapid/slow cutoff of $15 \mathrm{~km} \mathrm{~s}^{-1}$ for the remainder of the paper.

The overall disk fraction we obtain here is quite consistent with the disk fraction (at $24 \mu \mathrm{m}$ ) obtained elsewhere. A significant complication, however, within our sample and with comparison to other samples, is the ages of the stars, which we now discuss.

Slow rotators with disks are certainly in our sample. A possible explanation for the existence of slow rotators with excesses 
is long-lived pre-main-sequence (PMS) circumstellar disks. A number of theoretical models predict a strong correlation between rotation and primordial disk lifetime (via "disk locking"), whereby the angular rotational velocities of PMS stars are magnetically locked to the Keplerian velocity of the inner part of the star's disk as long as accretion continues (e.g., Königl 1991; Shu et al. 2000). Such correlations are observed through mid-IR excesses (taken to arise from primordial circumstellar dust) that are larger around slowly rotating PMS stars (e.g., Rebull et al. 2006; Kundurthy et al. 2006). These models predict that stars with long-lived accretion disks will arrive on the MS as slow rotators and those with short-lived accretion disks will arrive on the MS as rapid rotators. If one assumes that stars with long-lived accretion disks are more likely to form wellpopulated planetesimal belts, and therefore to have detectable debris disks at later ages, then there should be an anti-correlation between $v \sin i$ and $24 \mu \mathrm{m}$ excess for young low-mass MS stars. However, whether or not we see this correlation in our data set is certainly intimately tied to the issue of ages in these stars.

While Figure 1 demonstrates that there is no large, systematic, and obvious age difference between our rapid and slow rotators, it is almost certainly true that our red, rapidly rotating sample is younger-on average-than our red, slowly rotating sample. This follows simply from the fact that stars in the red sample $((B-V)>0.43)$ are expected to have dynamo-driven solartype winds and should thus spin down as they age. Slow rotators could either be stars (of any age) with long-lived PMS disks that arrived on the ZAMS as slow rotators or older stars that arrived on the MS as rapid rotators but subsequently lost angular momentum due to winds. Rapid rotators must necessarily be relatively young. Comparison to the rotational velocities of stars in open clusters in this color range-Pleiades, age $\sim 100 \mathrm{Myr}$ (Queloz et al. 1998); Hyades, age $~ 650$ Myr (Mermilliod et al. 2009, Figure 4); and M67, age 4 Gyr (Melo et al. 2001) - suggests that the red-rapid sample, and in particular the stars with excesses, probably has ages in the range $<100 \mathrm{Myr}$ to $\sim 1$ Gyr. (In fact, the sole blue-rapid rotator with a disk is also consistent with this younger age range, but with only one disk, the significance of this comparison is lessened.) See Figure 8 for the comparison to the Pleiades. This suggests that the red-rapid rotators are on average somewhat younger than the likely average age for the red-slow rotators, but not by a huge amount. A younger age for the red-rapid sample would help explain their higher $24 \mu \mathrm{m}$ excess frequency. We note that all of the stars with excesses have isochronal age estimates provided in Holmberg et al. (2009). The ages are all between 1.2 and $4.8 \mathrm{Gyr}$; the red-rapid sample makes up most of the disks, but is not distinctly younger than the rest of the objects. These ages seem at variance to the ages inferred from their rotational velocities. For these objects, Holmberg adopts metalpoor metallicities $(\langle[\mathrm{Fe} / \mathrm{H}]\rangle \approx-0.2)$, which may or may not be appropriate. Spectroscopic metallicity determinations for these stars would help considerably in the interpretation of their ages and hence the origin of their disks.

Nevertheless, the mere existence of significant debris disk excesses around the 11 members of the red-rapid sample is significant. Either the red-rapid sample includes a set of stars that are significantly younger than the rest of our sample (and the high disk frequency simply reflects the fact that younger stars more frequently have debris disks) or the red-rapid sample includes a set of stars of "normal" age but unusual dust content. Better ages for these stars would address the first possibility; $\mathrm{X}$-ray fluxes for this set of stars could help constrain whether

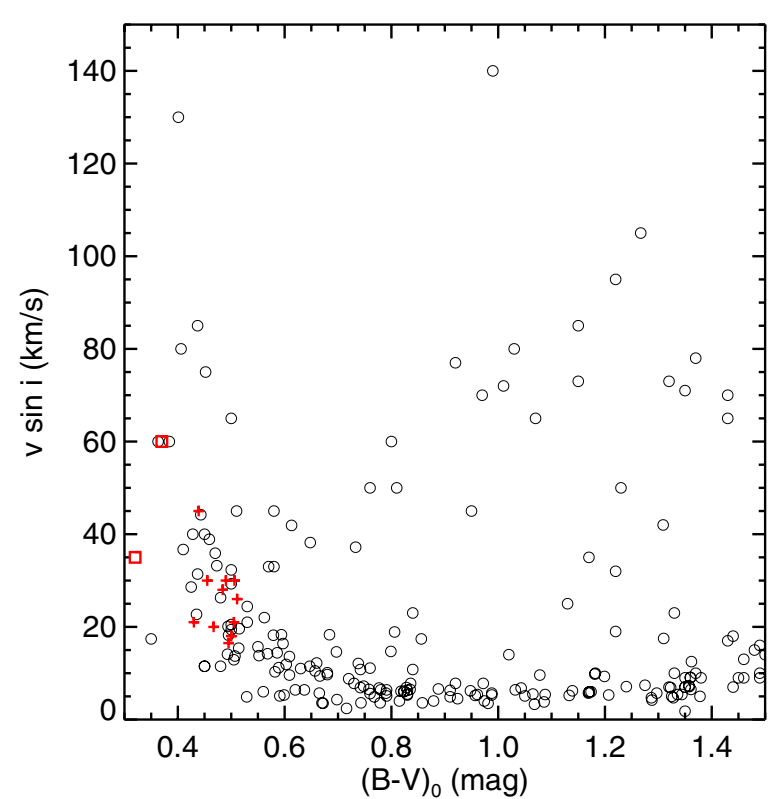

Figure 8. ( $B-V)_{0}$ vs. $v \sin i$ plot for the Pleiades (open circles), the eight redrapid rotators with disks from our analysis ( + signs), and, for completeness, the two blue-rapid rotators with an excess from our analysis (squares). The rapid rotators from our analysis have $v \sin i$ values consistent with those from "normal" F stars in the Pleiades.

(A color version of this figure is available in the online journal.)

they have unusually weak winds (for example) by use of the Wood et al. (2002) formula relating X-ray flux and mass loss rate for low-mass stars. Unfortunately, no X-ray fluxes are available for the red-rapid rotators with IR excesses, and hence we cannot estimate their mass loss rates using the Wood et al. (2002) formula. X-ray data for these stars and better age estimates (perhaps obtainable by combining Gaia parallaxes and improved $[\mathrm{Fe} / \mathrm{H}]$ estimates) could place interesting constraints on the dust production rates for these stars.

Sierchio et al. (2010), Stauffer et al. (2007), and Rebull et al. (2008) have all previously noted a possible correlation between $24 \mu \mathrm{m}$ excess and rotation, with excesses being rare or absent among rapid rotators. The samples of stars used in these studies have included F, G, and $\mathrm{K}$ dwarfs, and thus are on average of significantly later type than our sample. Even if the correlation with rotation in those samples is real (the statistical significance was not high), it does not necessarily contradict our result. The wind mass-loss rates from $\mathrm{G}$ and $\mathrm{K}$ dwarfs are likely much higher than for F dwarfs; the comparison is made difficult because direct measurements are not possible, and inferences from rotational velocities depend on the unknown degree to which the spin-down affects just the outer convective envelope or also the radiative core. Moreover, the relative strengths of wind and radiation pressure are important and F stars have higher luminosities than later-type stars (the ratio of mass loss to total luminosity is lower). If the $\mathrm{G}$ and $\mathrm{K}$ dwarf mass-loss rates are much higher, they could successfully scour their disks of dust and produce the apparent correlation, while the weaker winds of F dwarfs still allow debris dust.

One of our original goals was to determine if $\mathrm{F}$ dwarfs have a comparatively high debris disk frequency. By comparison to the FEPS GK stars of similar ages, our F stars have a comparable disk frequency. Another of our original goals was to look for evidence (via debris disk frequency) for development of winds at the F5 boundary where solar-type winds are expected to develop, and we do not see strong evidence of this. A larger sample of 
F stars would presumably reveal more disks, and thus enable better restrictions on the observed disk fraction. Observations in other infrared wavelength bands would also enable us to see if our $24 \mu \mathrm{m}$ excesses extend to longer or shorter wavelengths. A wider range of observations would allow for more conclusive evidence in understanding what is going on in these stars. It would also be of interest to compare our infrared observations with X-ray observations to see if there is any indication of strong activity, which would help to argue for or against the theory of Chen et al. (2005).

The authors wish to acknowledge truly helpful comments from the anonymous referee, and helpful conversations with Sidney Wolff.

This work is based in part on observations made with the Spitzer Space Telescope, which is operated by the Jet Propulsion Laboratory, California Institute of Technology under a contract with NASA. Support for this work was provided by NASA through an award issued by JPL/Caltech. The research described in this paper was partially carried out at the Jet Propulsion Laboratory, California Institute of Technology, under contract with the National Aeronautics and Space Administration. This research has made use of what was then called the NASA/IPAC/NExScI Star and Exoplanet Database, which was operated by the Jet Propulsion Laboratory, California Institute of Technology, under contract with the National Aeronautics and Space Administration.

This research has made use of NASA's Astrophysics Data System (ADS) Abstract Service, and of the SIMBAD database, operated at CDS, Strasbourg, France. This research has made use of data products from the Two Micron All Sky Survey (2MASS), which is a joint project of the University of Massachusetts and the Infrared Processing and Analysis Center, funded by the National Aeronautics and Space Administration and the National Science Foundation. This publication makes use of data products from the Wide-field Infrared Survey Explorer, which is a joint project of the University of California, Los Angeles, and the Jet Propulsion Laboratory/California Institute of Technology, funded by the National Aeronautics and Space Administration. The WISE and 2MASS data are served by the NASA/IPAC Infrared Science Archive, which is operated by the Jet Propulsion Laboratory, California Institute of Technology, under contract with the National Aeronautics and Space Administration. This research has made use of the Digitized Sky Surveys, which were produced at the Space Telescope Science Institute under U.S. Government grant NAG W-2166.

\section{APPENDIX}

\section{COMPARISON TO WISE}

This section explicitly considers a comparison of our photometric results at $24 \mu \mathrm{m}$ to those from WISE at $22 \mu \mathrm{m}$ (from the all-sky release from 2012 March). As seen in Figure 2, the ensemble of disk-free stars is distributed about $K_{s}-[24] \sim 0$, with a slightly blue offset. The scatter around $K_{s}-[24]=-0.03$ (once outliers are removed) is not very large. As seen in Figure 3, the ensemble of disk-free stars is distributed about $[3.4]-[22]=0$. Within WISE data, there is no offset, unlike what we found in $K_{s}-$ [24]. It is important to note that the $3.4 \mu \mathrm{m}$ (W1) detections here for the entire sample are saturated in the original data, and the values here are obtained via PSF fitting to the wings of the detection. The $22 \mu \mathrm{m}$ (W4) points are, in contrast, not saturated. (The WISE Explanatory Supplement

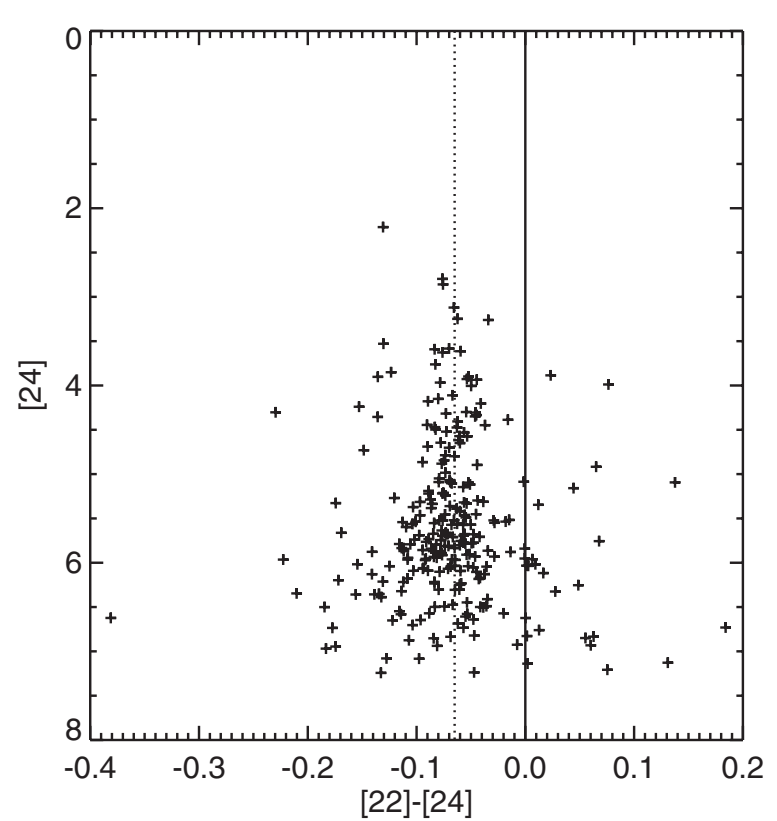

Figure 9. [24] vs. [22] - [24] for our sample. Note that the mean of the ensemble is $-0.065 \mathrm{mag}$. There is evidently an offset between the MIPS-24 zero point and the WISE-4 zero points for this sample.

states that the channels saturate at 8.1, 6.7, 3.8, and -0.4 mag for W1, W2, W3, and W4, respectively, but that fits to the unsaturated wings of the PSF allow viable magnitudes to be obtained up to $2.0,1.5,-3.0$, and -4 mag for the four channels, respectively.) A similar plot for this sample for W2 is not at all as clean, as per the Explanatory Supplement (because W2 is also saturated), but a similar plot for W3 is also very well behaved. Thus, the WISE data are quite internally consistent.

Figure 9 explicitly compares the WISE-4 $(22 \mu \mathrm{m})$ points and the MIPS-24 $(24 \mu \mathrm{m})$ points. In the ideal case, for plain photospheres, the measured Vega-based magnitude of a star should be the same at 22 and $24 \mu \mathrm{m}$. Since we have all F stars here, both 22 and $24 \mu \mathrm{m}$ are on the RJ side of the SED, and as such, the difference in magnitudes should be zero. As can be seen in Figure 9, it is not zero, but instead centered on $-0.065 \mathrm{mag}$. We wondered if this offset, while systematic, could be well within expected errors; however, $>95 \%$ of the ensemble of [22] errors for our sample as reported in the WISE catalog is less than $0.065 \mathrm{mag}$, and most of our MIPS-24 errors are $\sim 0.02$ mag. Our targets are from all over the sky, with a wide variety of backgrounds, but the backgrounds are still, on the whole, relatively low. The source density is not very high; the sources are largely unconfused in the MIPS data, and any source (e.g., apparent or actual binary, or background galaxy) close enough in projected distance to adversely affect the MIPS photometry should also affect the WISE photometry, in the same way. The MIPS photometry, as well as the WISE photometry, is obtained via PSF fitting. The WISE photometry is calibrated primarily from disk-free A stars; while color corrections are required for the WISE photometry for very red sources, our targets should not require such corrections. The WISE team uses a different zero point for MIPS-24; they use 7.449 Jy rather than the $7.17 \mathrm{Jy}$ we used. The value we use comes from the MIPS Instrument Handbook. If we instead use $7.449 \mathrm{Jy}$, this worsens the discrepancy seen in Figure 9.

Figure 10 shows $K_{s}$ versus $K_{s}-$ [22] for our sample. As in Figure 9 above, there is an offset of 0.065 mag. Section VI.3.i.4 


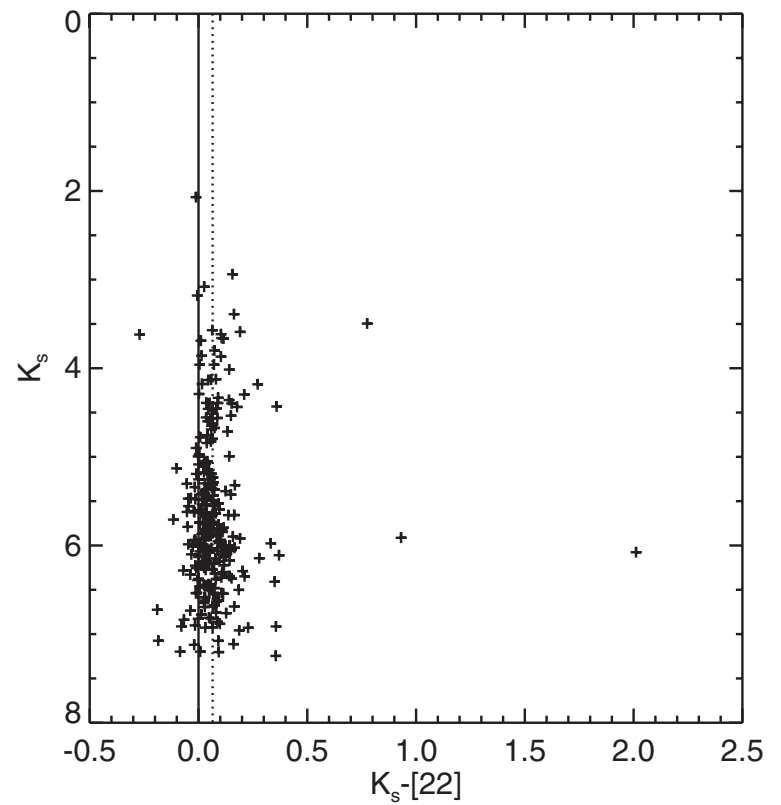

Figure 10. $K_{s}$ vs. $K_{s}-$ [22] for our sample. Note that the mean of the ensemble is not zero, but instead +0.065 (dotted line).

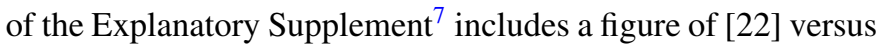
$K_{s}-$ [22] for a cluster of stars; there an offset can be seen in $K_{s}-[22]$ that is comparable to, or even a little larger than, what we measure. So, there is a known offset between $K_{s}$ and [22]. Since our [24] measurements are well matched to $K_{s}$ (as per Figure 2), there is also an offset between [22] and [24]; for our F star sample, this is $\sim 0.065 \mathrm{mag}$.

Absolute calibrations are difficult. While the WISE absolute calibration may be correct in the end, the MIPS $24 \mu \mathrm{m}$ calibration is closer to the 2MASS $K_{S}$ calibration, at least for our MIPS reduction and our largely disk-free F star sample.

\section{REFERENCES}

Beichman, C., Bryden, G., Stapelfeldt, K. R., et al. 2006, ApJ, 652, 1674 Bessell, M. S. 2000, PASP, 112, 961

Bouvier, J., Forestini, M., \& Allain, S. 1997, A\&A, 326, 1023

Burgasser, A. J., Kirkpatrick, J. D., Reid, I. N., et al. 2003, ApJ, 586, 512 Carpenter, J. M., Bouwman, J., Mamajek, E. E., et al. 2009, ApJS, 181, 197

Carpenter, J. M., Bouwman, J., Silverstone, M. D., et al. 2008, ApJS, 179, 423 Chen, C. H., Jura, M., Gordon, K. D., \& Blaylock, M. 2005, ApJ, 623, 493

Cieza, L., Cochran, W., \& Augereau, J.-C. 2008, ApJ, 679, 720

Currie, T., Lada, C. J., Plavchan, P., et al. 2009, ApJ, 698, 1

Cutri, R., Skrutskie, M. F., Van Dyk, S., et al. 2003, Explanatory Supplement to the 2MASS All Sky Data Release and Extended Mission Products, http://www.ipac.caltech.edu/2mass/releases/allsky/doc/explsup.html

Denissenkov, P., Pinsonneault, M., Terndrup, D., \& Newsham, G. 2010, ApJ, 716,1269
Engelbracht, C. W., Blaylock, M., Su, K. Y. L., et al. 2007, PASP, 119 994

Gautier, T. N., Rieke, G. H., Stansberry, J., et al. 2007, ApJ, 667, 527

Guenther, E., Hartmann, M., Esposito, M., et al. 2009, A\&A, 507, 1659

Holmberg, J., Nordstrom, B., \& Andersen, J. 2007, A\&A, 475, 519

Holmberg, J., Nordström, B., \& Andersen, J. 2009, A\&A, 501, 941

Huang, S.-S. 1965, ApJ, 141, 985

Huchra, J., Pughe, W., Kleinmann, S., et al. 1994, in ASP Conf. Ser. 67, Unveiling Large-Scale Structures Behind the Milky Way, ed. C. Balkowski \& R. Kraan (San Francisco, CA: ASP), 41

Kalas, P., Graham, J., Clampin, M., \& Fitzgerald, M. 2006, ApJ, 637, L57

Kenyon, S. J., \& Hartmann, L. 1995, ApJS, 101, 117

Koerner, D., Kim, S., Trilling, D. E., et al. 2010, ApJ, 710, L26

Königl, A. 1991, ApJ, 370, L39

Kundurthy, P., Meyer, M. R., Robberto, M., Beckwith, S. V. W., \& Herbst, T. 2006, AJ, 132, 2469

Lawler, S., Beichman, C. A., Bryden, G., et al. 2009, ApJ, 705, 89

Makovoz, D., \& Marleau, F. R. 2005, PASP, 117, 1113

Melo, C. H. F., Pasquini, L., \& De Medeiros, J. R. 2001, A\&A, 375, 851

Mermilliod, J.-C., Mayor, M., \& Udry, S. 2009, A\&A, 498, 949

Mestel, L., \& Roxburgh, I. W. 1962, ApJ, 136, 615

Meyer, M., Carpenter, J. M., Mamajek, E. E., et al. 2008, ApJ, 673, L181

Meyer, M., Hillenbrand, L. A., Backman, D., et al. 2006, PASP, 118 , 1690

Moór, A., Pascucci, I., Kóspál, Á., et al. 2011, ApJS, 193, 4

Nordstrom, B., Mayor, M., Andersen, J., et al. 2004, A\&A, 418, 989

Papovich, C., Dole, H., Egami, E., et al. 2004, ApJS, 154, 70

Plavchan, P., Jura, M., \& Lipscy, S. J. 2005, ApJ, 631, 1161

Plavchan, P., Werner, M. W., Chen, C. H., et al. 2009, ApJ, 698, 1068

Queloz, D., Allain, S., Mermilliod, J.-C., Bouvier, J., \& Mayor, M. 1998, A\&A, 335,183

Rebull, L. M., Padgett, D. L., McCabe, C.-E., et al. 2010, ApJS, 186, 259

Rebull, L. M., Stapelfeldt, K. R., Werner, M. W., et al. 2008, ApJ, 681, 1484

Rebull, L. M., Stauffer, J. R., Megeath, S. T., Hora, J. L., \& Hartmann, L. 2006, ApJ, 646, 297

Rhee, J. H., Song, I., Zuckerman, B., \& McElwain, M. 2007, ApJ, 660, 1556

Rieke, G., Blaylock, M., Decin, L., et al. 2008, AJ, 135, 224

Rieke, G., Young, E. T., Engelbracht, C. W., et al. 2004, ApJS, 154, 25

Schröder, C., Reiners, A., \& Schmitt, J. H. M. M. 2009, A\&A, 493, 1099

Shu, F., Najita, J. R., Shang, H., \& Li, Z.-Y. 2000, in Protostars and Planets IV, ed. V. Mannings, A. P. Boss, \& S. S. Russell (Tucson, AZ: Univ. Arizona Press), 789

Sierchio, J. M., Rieke, G. H., Su, K. Y. L., et al. 2010, ApJ, 712, 1421

Sills, S., Pinsonneault, M., \& Terndrup, D. 2000, ApJ, 534, 335

Simon, T., \& Landsman, W. 1991, ApJ, 380, 200

Skrutskie, M., Cutri, R. M., Stiening, R., et al. 2006, AJ, 131, 1163

Stauffer, J., Rebull, L. M., Carpenter, J., et al. 2005, AJ, 130, 1834

Stauffer, J., et al. 2007, in IAU Symp. 243, Star-Disk Interaction in Young stars, Poster Abstracts

Stencel, R. E., \& Backman, D. E. 1991, ApJS, 75, 905

Su, K., Rieke, G. H., Stansberry, J. A., et al. 2006, ApJ, 653, 675

Trilling, D., Bryden, G., Beichman, C., et al. 2008, ApJ, 674, 1086

Trilling, D., Stansberry, J. A., Stapelfeldt, K. R., et al. 2007, ApJ, 658, 1289

Urban, L., Rieke, G., Su, K., \& Trilling, D. 2012, ApJ, 750, 98

Werner, M. W., Roellig, T. L., Low, F. J., et al. 2004, ApJS, 154, 1

Winn, J., Fabrycky, D., Albrecht, S., \& Johnson, J. A. 2010, ApJ, 718, L145

Wolff, S., \& Simon, T. 1997, PASP, 109, 759

Wood, B., Müller, H.-R., Zank, G., \& Linsky, J. 2002, ApJ, 574, 412

Wright, E., Eisenhardt, P. R. M., Mainzer, A. K., et al. 2010, AJ, 140, 1868

Wyatt, M. 2005, A\&A, 433, 1007

Zuckerman, B., Rhee, J., Song, I., \& Bessell, M. 2011, ApJ, 732, 61

\footnotetext{
7 Specifically, http://WISE2.ipac.caltech.edu/docs/release/allsky/
} expsup/sec6_3c.html\#measure_bias. 